Supporting Information

A DFT Study on the Electrocatalytic Reduction of $\mathrm{CO}_{2}$ to $\mathrm{CO}$

by a Molecular Chromium Complex

Juan J. Moreno, Shelby L. Hooe, Charles W. Machan*

Department of Chemistry, University of Virginia, McCormick Road PO Box 400319, Charlottesville, VA 22904-4319, United States

JJM ORCID: 0000-0003-1809-6170; SLH ORCID: 0000-0002-6991-2273

*Correspondence to: machan@virginia.edu; ORCID: 0000-0002-5182-1138 


\section{Use of $\mathrm{Fc}^{+} / \mathrm{Fc}$ as Computational Reference}

In agreement with a previous report, ${ }^{1}$ our computation of redox potentials relative to $\mathrm{Fc}^{+} / \mathrm{Fc}$ with B3LYP gave an excellent correlation between calculated and experimental data $\left(\mathrm{R}^{2}=0.994\right)$, but computed values were systematically more positive $(0.50 \mathrm{~V}$ in average, $c f$. the $0.48 \mathrm{~V}$ value found by Batista and coworkers with $\left.\mathrm{R}^{2}=0.96\right)$. However, this underestimation does not occur when a different reference pair is considered: we employed the phenazine $\mathrm{PHNZ}^{-} / \mathrm{PHNZ}$ pair $(\mathrm{PHNZ}=$ phenazine) and referenced to $\mathrm{Fc}^{+} / \mathrm{Fc}$ values from experimental data $\left(\mathrm{E}_{\mathrm{PHNZ}-/ \mathrm{PHNZ}}=-1.63 \mathrm{~V}\right.$ in DMF, see Table 1 and Figure S8).

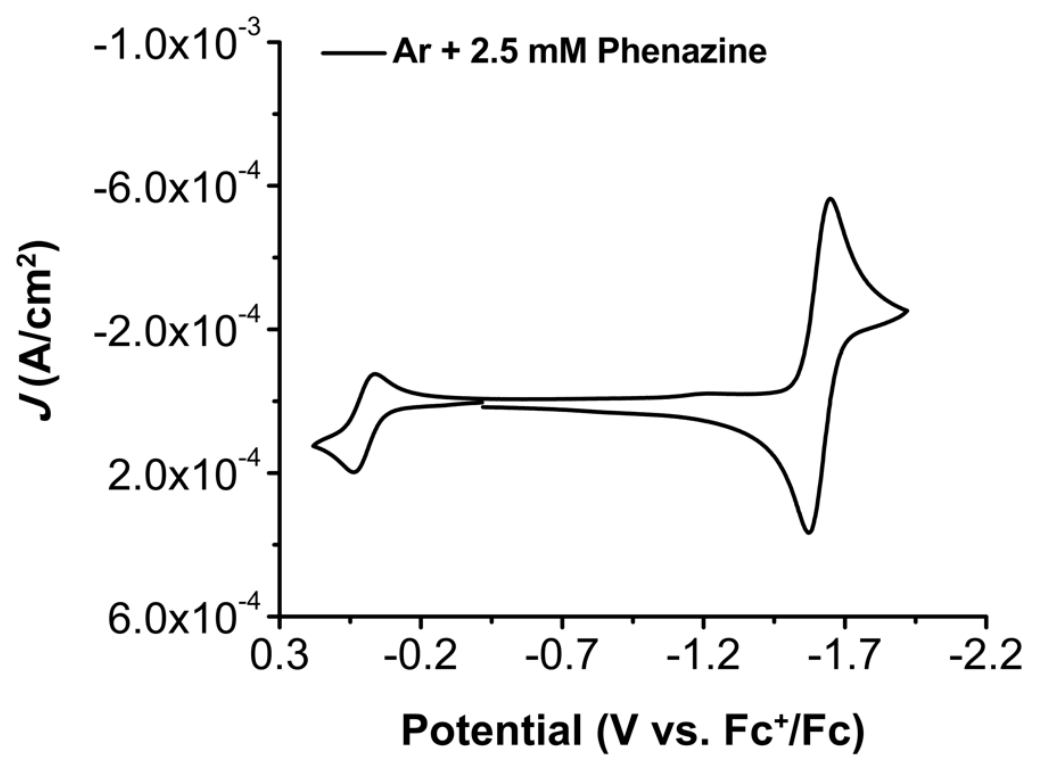

Figure S1. CVs of $2.5 \mathrm{mM}$ phenazine (PHNZ) under argon (Ar) saturation conditions. Conditions: $0.1 \mathrm{M} \mathrm{TBAPF} / \mathrm{DMF}$; glassy carbon working electrode, glassy carbon rod counter electrode, $\mathrm{Ag} / \mathrm{AgCl}$ pseudoreference electrode; $100 \mathrm{mV} / \mathrm{s}$ scan rate; referenced to internal ferrocene standard. 


\section{Chloride-Displacement Equilibrium}

Table S1. Relative potential (E) and corrected (concentration and quasiharmonic) triple zeta free energies $([\mathrm{qG}])$ for $\mathrm{Cr}(\mathrm{L}) \mathrm{Cl}(\mathrm{DMF})_{\mathrm{n}}(\mathrm{n}=0,1)$ species, in $\mathrm{kcal} / \mathrm{mol}$.

\begin{tabular}{|c|c|c|c|}
\hline Species & $\mathbf{E}$ & {$[\mathbf{q G}]$} & $<\mathbf{S}^{\mathbf{2}} \mathbf{( \mathbf { D Z } / \mathbf { T Z } )}$ \\
\hline${ }_{0}^{2} \mathbf{C r}(\mathbf{C l})^{0}+\mathrm{DMF}$ & +41.5 & +29.4 & $0.7735 / 0.7714$ \\
\hline${ }_{0}^{4} \mathbf{C r}(\mathbf{C l})^{0}+\mathrm{DMF}$ & +19.8 & +7.2 & $3.7503 / 3.7503$ \\
\hline${ }_{1}^{2} \mathbf{C r}(\mathbf{C l})^{0}$ & +21.6 & +21.9 & $0.7708 / 0.7716$ \\
\hline${ }_{1}^{4} \mathbf{C r}(\mathbf{C l})^{0}$ & 0.0 & 0.0 & $3.7502 / 3.7502$ \\
\hline
\end{tabular}

Table S2. Relative potential (E) and corrected (concentration and quasiharmonic) triple zeta free energies $([\mathrm{qG}])$ for $\left[\mathrm{Cr}(\mathrm{L})(\mathrm{DMF})_{2}\right]^{+}$species, in $\mathrm{kcal} / \mathrm{mol}$.

\begin{tabular}{|l|c|c|c|}
\hline Species & $\mathbf{E}$ & {$[\mathbf{q G}]$} & $<\mathbf{S}^{2}>(\mathbf{D Z} / \mathbf{T Z})$ \\
\hline${ }_{2}^{2} \mathbf{C r}^{+1}$ & +24.4 & +25.0 & $0.7663 / 0.7669$ \\
\hline${ }_{2}^{4} \mathbf{C r}^{+1}$ & 0.0 & 0.0 & $3.7501 / 3.7501$ \\
\hline
\end{tabular}

Table S3. Relative potential (E) and corrected (concentration and quasiharmonic) triple zeta free energies $([\mathrm{qG}])$ comparing $\mathrm{Cr}(\mathrm{L}) \mathrm{Cl}(\mathrm{DMF})$ and $\left[\mathrm{Cr}(\mathrm{L})(\mathrm{DMF})_{2}\right]^{+}$species, in $\mathrm{kcal} / \mathrm{mol}$.

\begin{tabular}{|c|c|c|c|}
\hline Species & $\mathbf{E}$ & [qG] & $\left.<\mathbf{S}^{2}>\mathbf{( D Z} / \mathbf{T Z}\right)$ \\
\hline${ }_{0}^{4} \mathbf{C r}(\mathbf{C l})^{0}+\mathrm{DMF}$ & +4.8 & +2.9 & Table S2 \\
\hline${ }_{2}^{4} \mathbf{C r}^{+1}+\mathrm{Cl}^{-}$ & 0.0 & 0.0 & Table S2 \\
\hline${ }_{0}^{4} \mathbf{C r}(\mathbf{C l})^{0} \cdot \mathrm{DMF}$ & -1.5 & +7.7 & $3.7502 / 3.7502$ \\
\hline${ }_{2}^{4} \mathbf{C r}^{+1} \cdot \mathrm{Cl}^{-}$ & -3.6 & +7.0 & $3.7501 / 3.7501$ \\
\hline
\end{tabular}




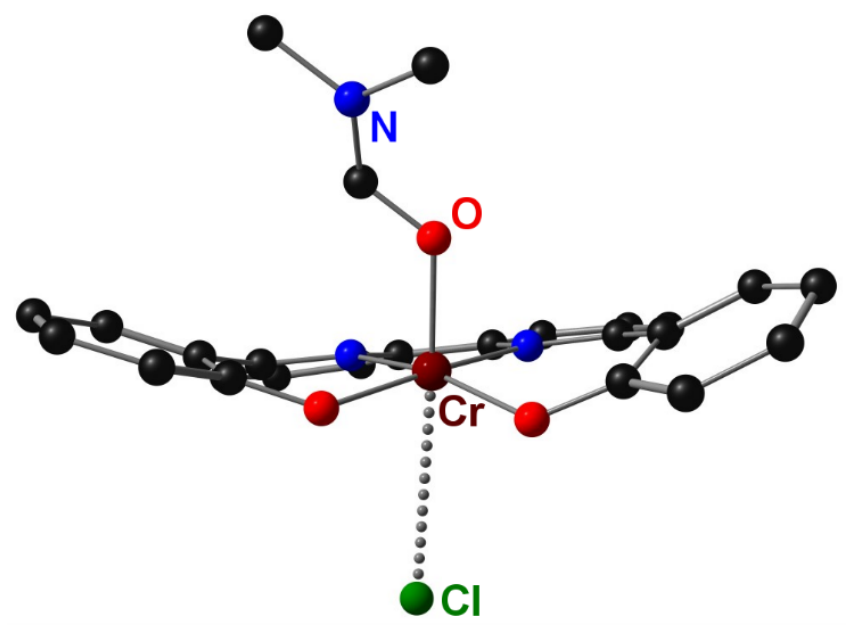

Figure S2. Molecular geometry of the transition state $\left({ }_{1}^{4} \mathbf{T S 1}{ }^{0},+15.0 \mathrm{kcal} / \mathrm{mol}\right)$ for the dissociation of chloride from ${ }_{1}^{4} \mathbf{C r}(\mathbf{C l}){ }^{0}$. H atoms and tBu groups omitted for clarity. 


\section{Reduction Pathways to $\mathrm{CO}_{2}$ Binding}

Table S4. Relative potential (E) and corrected (concentration and quasiharmonic) triple zeta free energies $([\mathrm{qG}])$ for $\operatorname{Cr}(\mathrm{L})(\mathrm{DMF})_{\mathrm{n}}(\mathrm{n}=0-2)$ species, in $\mathrm{kcal} / \mathrm{mol}$. Entries in red presented large spin contamination.

\begin{tabular}{|c|c|c|c|}
\hline Species & $\mathbf{E}$ & {$[\mathbf{q G}]$} & $<\mathbf{S}^{2}>(\mathbf{D Z} / \mathbf{T Z})$ \\
\hline${ }_{0}^{1} \mathbf{C r}^{0}+2 \mathrm{DMF}$ & +37.5 & +39.2 & $3.7839 / 3.7906$ \\
\hline${ }_{0}^{3} \mathbf{C r}^{0}+2 \mathrm{DMF}$ & +25.8 & +26.4 & $2.0234 / 2.0240$ \\
\hline${ }_{0}^{5} \mathbf{C r}^{0}+2 \mathrm{DMF}$ & 0.0 & 0.0 & $6.0001 / 6.0001$ \\
\hline${ }_{1}^{1} \mathbf{C r}^{0}+\mathrm{DMF}$ & +19.8 & +33.6 & $2.1874 / 2.3131$ \\
\hline${ }_{1}^{3} \mathbf{C r}^{0}+\mathrm{DMF}$ & -2.3 & +10.4 & $2.0299 / 2.0309$ \\
\hline${ }_{1}^{5} \mathbf{C r}^{0}+\mathrm{DMF}$ & -10.3 & +1.2 & $6.0001 / 6.0001$ \\
\hline${ }^{1} \mathbf{C r}{ }^{0}$ & +3.4 & +29.1 & $2.5273 / 2.6544$ \\
\hline${ }^{3} \mathbf{C r}{ }^{0}$ & -21.5 & +3.8 & $2.0329 / 2.0337$ \\
\hline${ }^{5} \mathbf{C r}{ }^{0}$ & -16.5 & +7.9 & $6.0002 / 6.0002$ \\
\hline
\end{tabular}

Redox Potential Determination

$$
\mathrm{PHNZ}^{-}+{ }_{2}^{4} \mathrm{Cr}^{+1} \rightleftharpoons \mathrm{PHNZ}+{ }_{0}^{5} \mathrm{Cr}^{0}+2 \mathrm{DMF}
$$

Eq S1: $\Delta[\mathrm{qG}]=1.6 \mathrm{kcal} / \mathrm{mol}$

$\mathrm{V}$ vs $\mathrm{PHNZ}^{-} / \mathrm{PHNZ}=-0.07 . \mathrm{V}$ vs $\mathrm{Fc}^{+} / \mathrm{Fc}=-1.63$. 
A
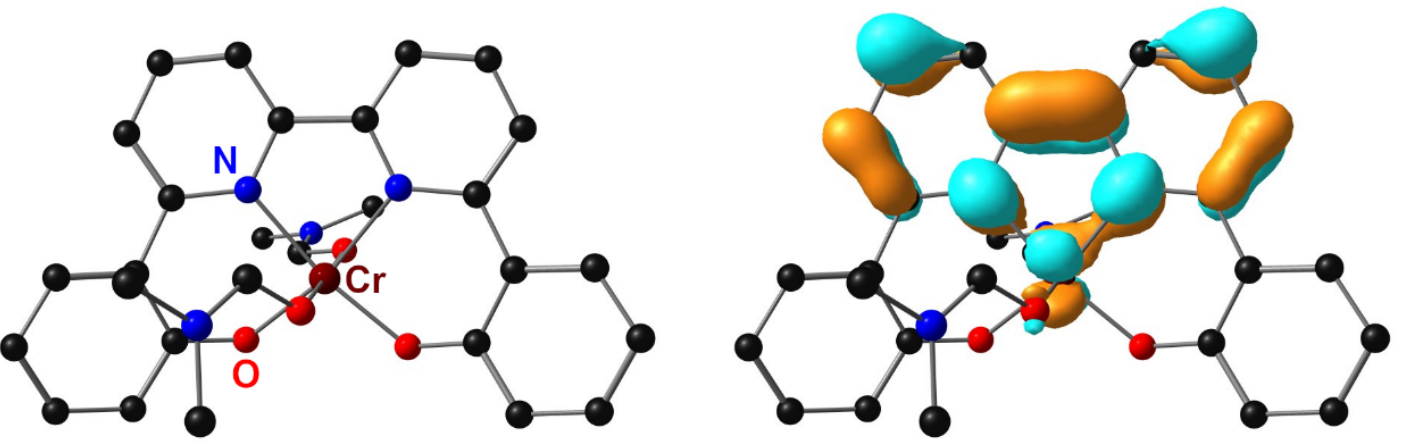

B
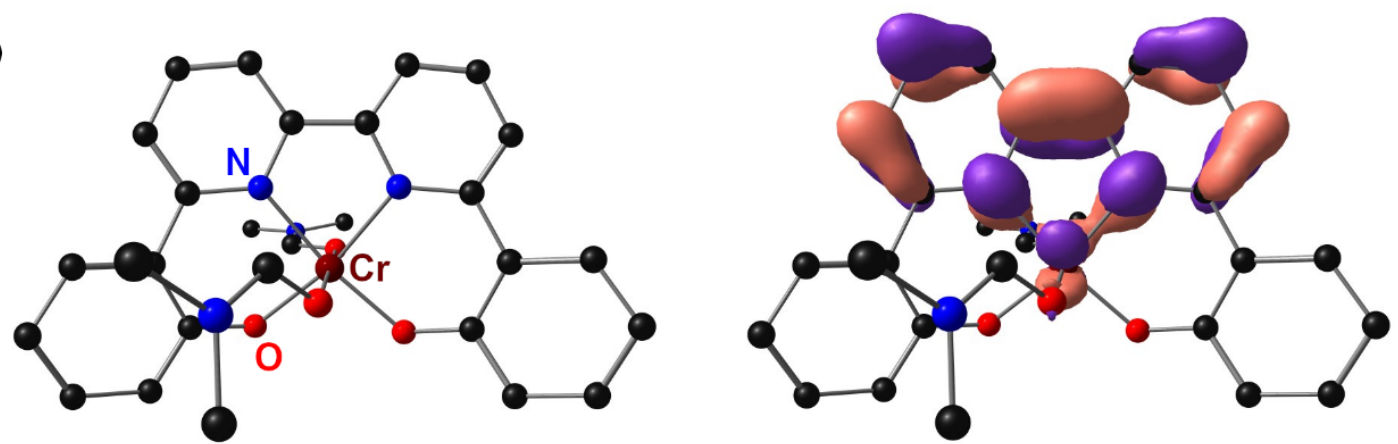

Figure S3. (A) LUMO of ${ }_{2}^{4} \mathbf{C r}^{+1}$, (B) highest SOMO of ${ }_{2}^{3} \mathbf{C r}^{0}$. H atoms and tBu groups omitted for clarity.
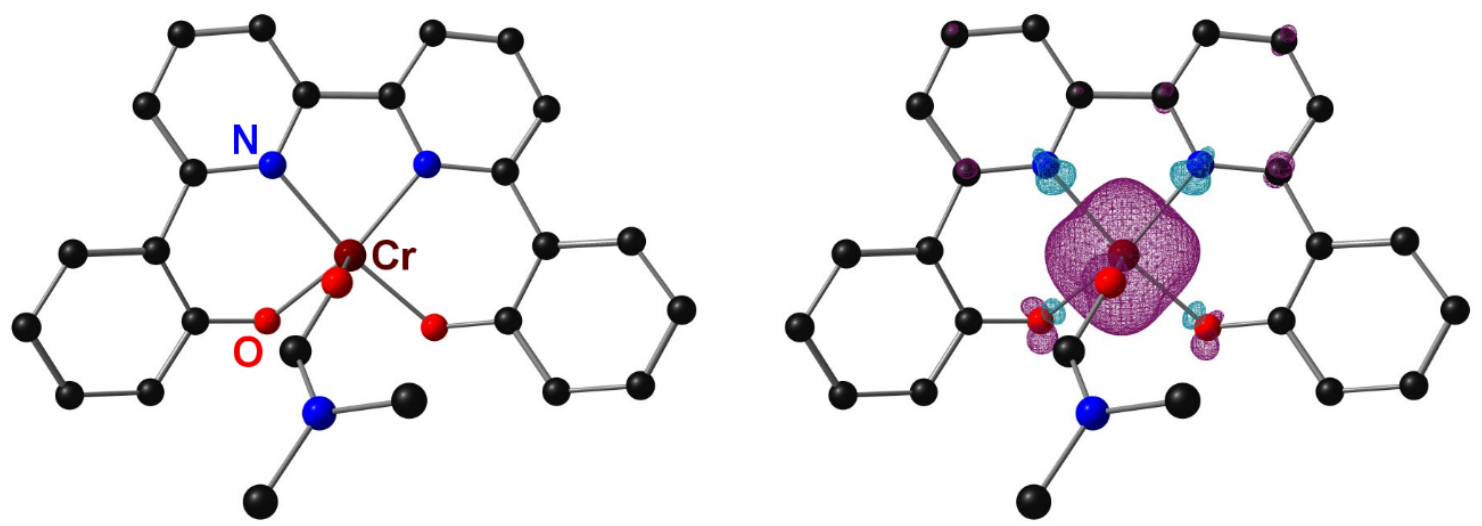

Figure S4. Spin density plot of ${ }_{1}^{5} \mathbf{C r}^{0}$. H atoms and tBu groups omitted for clarity. 
Table S5. Relative potential (E) and corrected (concentration and quasiharmonic) triple zeta free energies $([\mathrm{qG}])$ for $\mathrm{Cr}(\mathrm{L})(\mathrm{Cl})(\mathrm{DMF})_{\mathrm{n}}(\mathrm{n}=0,1)$ species, in $\mathrm{kcal} / \mathrm{mol}$. Entries in red presented large spin contamination.

\begin{tabular}{|c|c|c|c|}
\hline Species & $\mathbf{E}$ & [qG] & $<\mathrm{S}^{2}>(\mathrm{DZ} / \mathrm{TZ})$ \\
\hline${ }_{0}^{1} \mathrm{Cr}(\mathrm{Cl})^{-1}+\mathrm{DMF}$ & +31.2 & +33.1 & $2.521 / 2.5831$ \\
\hline${ }_{0}^{3} \mathrm{Cr}(\mathrm{Cl})^{-1}+\mathrm{DMF}$ & +7.0 & +7.6 & $2.0422 / 2.0428$ \\
\hline${ }_{0}^{5} \mathrm{Cr}(\mathrm{Cl})^{-1}+\mathrm{DMF}$ & 0.0 & 0.0 & $6.0001 / 6.0001$ \\
\hline${ }_{1}^{1} \mathrm{Cr}(\mathrm{Cl})^{-1}$ & +14.6 & +28.4 & $2.6967 / 2.8643$ \\
\hline${ }_{1}^{3} \mathrm{Cr}(\mathrm{Cl})^{-1}$ & -8.4 & +4.4 & $2.0398 / 2.0405$ \\
\hline${ }_{1}^{5} \mathrm{Cr}(\mathrm{Cl})^{-1}$ & -4.0 & +8.3 & $6.0003 / 6.0003$ \\
\hline
\end{tabular}

$$
P H N Z^{-}+{ }_{1}^{4} \operatorname{Cr}(\mathrm{Cl})^{0} \rightleftharpoons P H N Z+{ }_{0}^{5} \operatorname{Cr}(\mathrm{Cl})^{-1}+D M F
$$

Eq S2: $\Delta[\mathrm{qG}]=5.1 \mathrm{kcal} / \mathrm{mol}$ $\mathrm{V}$ vs $\mathrm{PHNZ}^{-} / \mathrm{PHNZ}=-0.22 . \mathrm{V}$ vs Fc$/ \mathrm{Fc}=-1.76$.

\section{A}
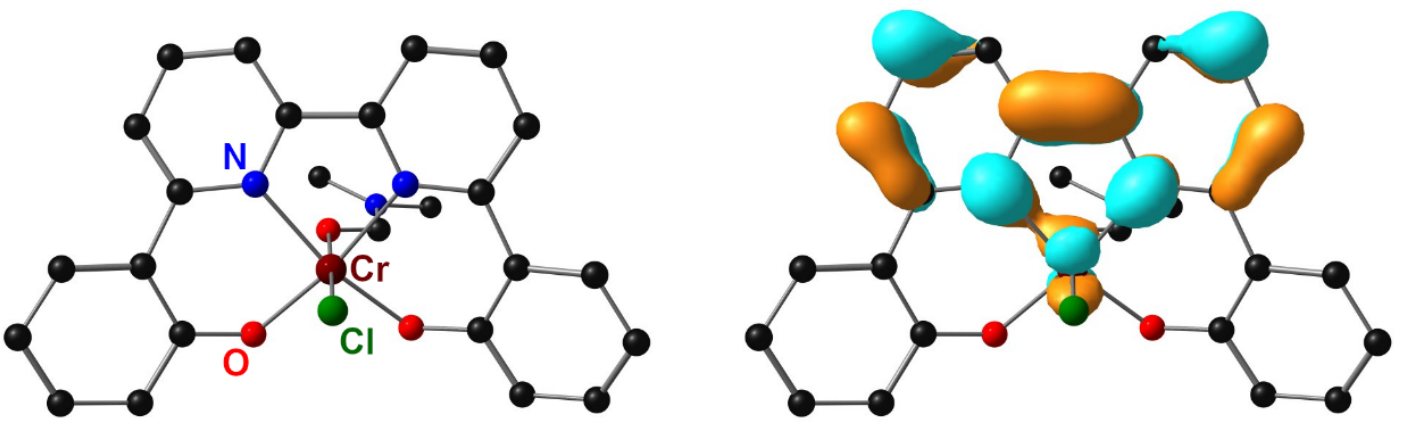

B
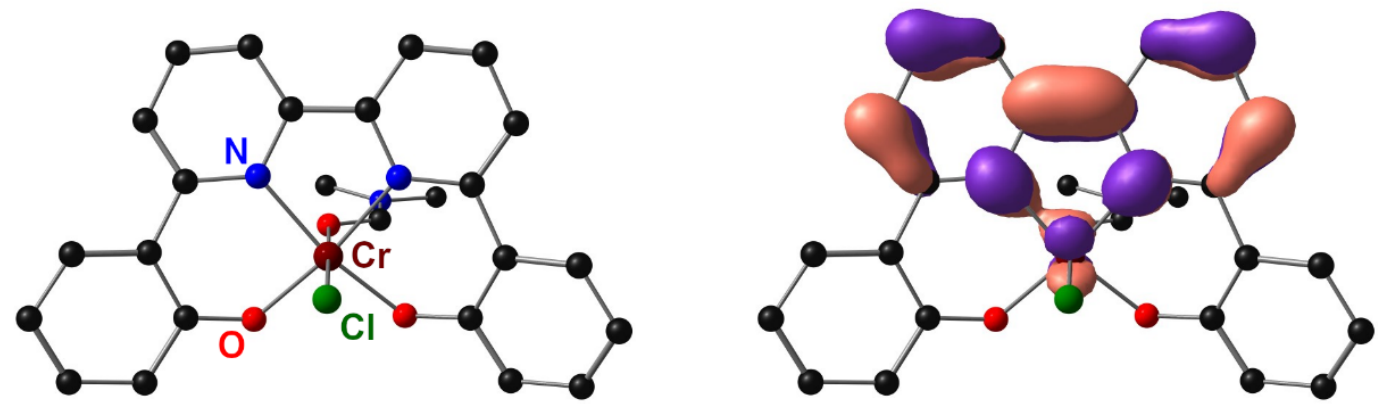

Figure S5. (A) LUMO of ${ }_{1}^{4} \mathbf{C r}(\mathbf{C l})^{0}$, (B) highest SOMO of ${ }_{1}^{3} \mathbf{C r}(\mathbf{C l})^{-1}$. H atoms and $\mathrm{tBu}$ groups omitted for clarity. 
Table S6. Relative potential (E) and corrected (concentration and quasiharmonic) triple zeta free energies $([\mathrm{qG}])$ for the transition states for chloride dissociation from ${ }_{0}^{5} \mathbf{C r}(\mathbf{C l})^{-1}$, in kcal $/ \mathrm{mol}$.

\begin{tabular}{|c|c|c|c|}
\hline Species & $\mathbf{E}$ & [qG] & $<\mathrm{S}^{2}>(\mathrm{DZ} / \mathrm{TZ})$ \\
\hline${ }_{0}^{3} \mathbf{T S 2}^{-1}+\mathrm{DMF}$ & +27.9 & +28.7 & $2.0227 / 2.0233$ \\
\hline${ }_{0}^{5} \mathbf{T S 2}^{-1}+\mathrm{DMF}$ & +2.9 & +3.5 & $6.0001 / 6.0001$ \\
\hline${ }_{1}^{3}$ TS2 $^{-1}$ & +0.9 & +14.0 & $2.0289 / 2.0298$ \\
\hline${ }_{1}^{5}$ TS2 $^{-1}$ & +0.6 & +11.2 & $6.0002 / 6.0002$ \\
\hline
\end{tabular}

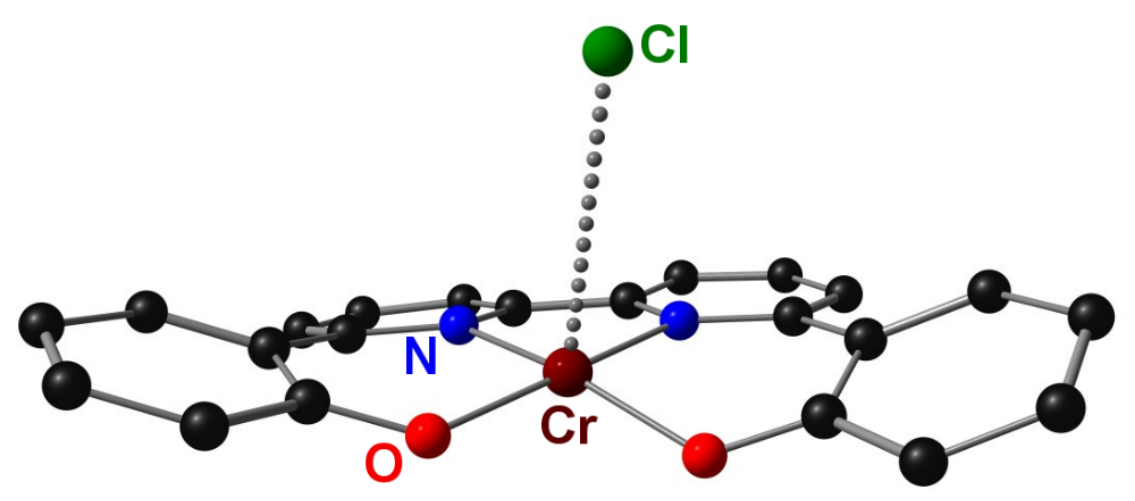

Figure S6. Molecular geometry of the transition state $\left({ }_{0}^{5} \mathbf{T S 2}^{-1},+3.5 \mathrm{kcal} / \mathrm{mol}([\mathrm{qG}))\right.$ for the dissociation of chloride from ${ }_{0}^{5} \mathbf{C r}(\mathbf{C l})^{-1}$. H atoms and tBu groups omitted for clarity.

$$
{ }_{0}^{5} \mathrm{Cr}(\mathrm{Cl})^{-1} \rightleftharpoons{ }_{0}^{5} \mathrm{Cr}^{0}+\mathrm{Cl}^{-}
$$

Eq S3: $\Delta[\mathrm{qG}]=-6.4 \mathrm{kcal} / \mathrm{mol}$ 
A
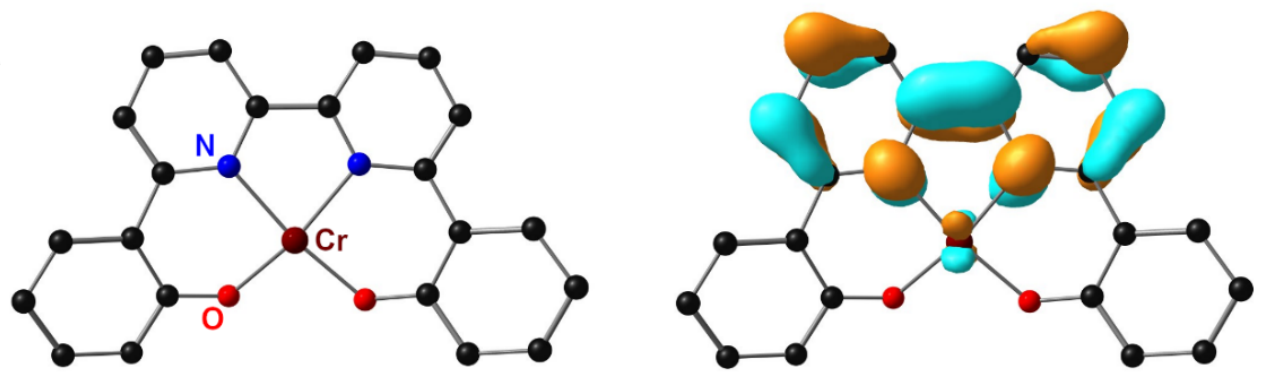

B
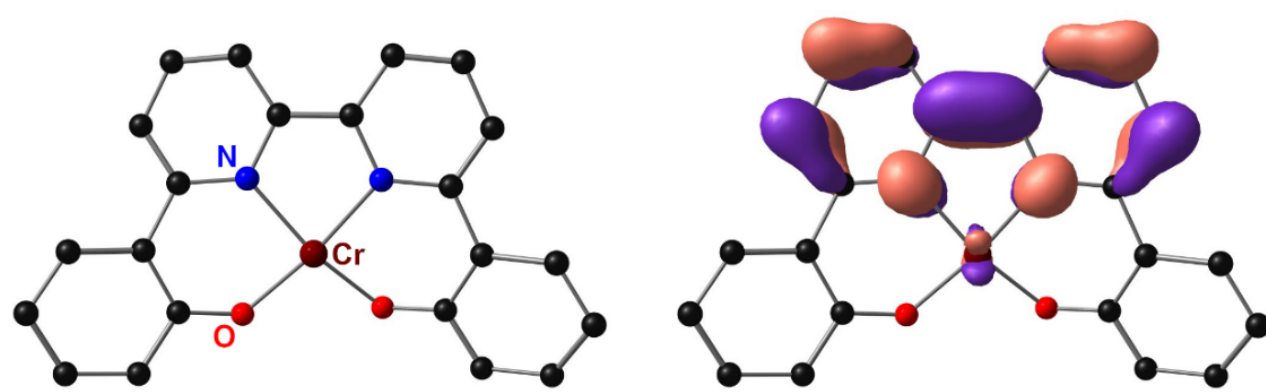

Figure S7. (A) LUMO of ${ }_{0}^{5} \mathbf{C r}^{0}$, (B) highest SOMO of ${ }_{0}^{4} \mathrm{Cr}^{-1}$. H atoms and tBu groups omitted for clarity.

Table S7. Relative potential (E) and corrected (concentration and quasiharmonic) triple zeta free energies $([\mathrm{qG}])$ for $\left[\mathrm{Cr}(\mathrm{L})(\mathrm{DMF})_{\mathrm{n}}\right]^{-}(\mathrm{n}=0-2)$ species, in $\mathrm{kcal} / \mathrm{mol} .{ }_{2}^{6} \mathrm{Cr}^{-1}$ spontaneously releases a DMF molecule. Entries in red presented large spin contamination.

\begin{tabular}{|c|c|c|c|}
\hline Species & $\mathbf{E}$ & {$[\mathbf{q G}]$} & $\left.<\mathbf{S}^{2}>\mathbf{( D Z} / \mathbf{T Z}\right)$ \\
\hline${ }_{0}^{2} \mathbf{C r}^{-1}+2 \mathrm{DMF}$ & +24.8 & +25.6 & $3.0895 / 3.1089$ \\
\hline${ }_{0}^{4} \mathbf{C r}^{-1}+2 \mathrm{DMF}$ & 0.0 & 0.0 & $3.7751 / 3.7756$ \\
\hline${ }_{0}^{6} \mathbf{C r}^{-1}+2 \mathrm{DMF}$ & +4.3 & +3.8 & $8.7501 / 8.7501$ \\
\hline${ }_{1}^{2} \mathbf{C r}^{-1}+\mathrm{DMF}$ & +15.7 & +27.6 & $3.1805 / 3.1943$ \\
\hline${ }_{1}^{4} \mathbf{C r}^{-1}+\mathrm{DMF}$ & -9.7 & +1.5 & $3.7768 / 3.7772$ \\
\hline${ }_{1}^{6} \mathbf{C r}^{-1}+$ DMF & -5.6 & +5.2 & $8.7501 / 8.7501$ \\
\hline${ }_{2}^{2} \mathbf{C r}^{-1}$ & -0.3 & +23.6 & $2.8000 / 2.8559$ \\
\hline${ }_{2}^{4} \mathbf{C r}^{-1}$ & -7.1 & +16.9 & $3.7518 / 3.7517$ \\
\hline
\end{tabular}




$$
P H N Z^{-}+{ }_{0}^{5} \mathrm{Cr}^{0} \rightleftharpoons P H N Z+{ }_{0}^{4} \mathrm{Cr}^{-1}
$$

Eq S4: $\Delta[\mathrm{qG}]=11.4 \mathrm{kcal} / \mathrm{mol}$

$\mathrm{V}$ vs $\mathrm{PHNZ}^{-} / \mathrm{PHNZ}=-0.50 . \mathrm{V}$ vs $\mathrm{Fc}^{+} / \mathrm{Fc}=-2.01$.
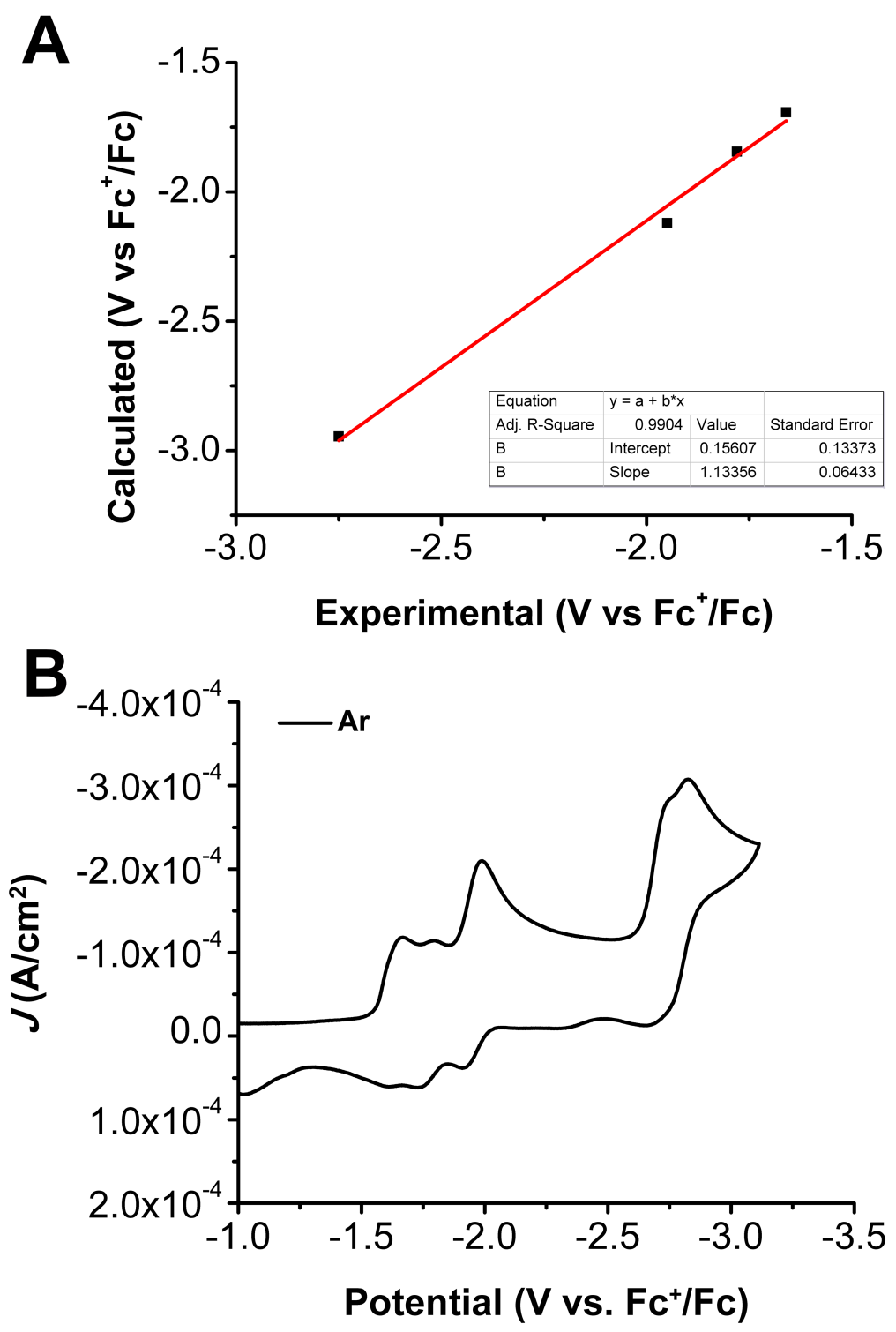

Figure S8. (A) Plot of calculated versus experimental redox potentials at the triple-zeta level, (B) $\mathrm{CV}$ of $\mathrm{Cr}\left({ }^{\text {tbu }} \mathrm{dhbpy}\right) \mathrm{Cl}\left(\mathrm{H}_{2} \mathrm{O}\right)$ under Ar saturation conditions. Conditions: 1.0 $\mathrm{mM}$ analyte with $0.1 \mathrm{M} \mathrm{TBAPF} / \mathrm{DMF}$; glassy carbon working electrode, glassy carbon rod counter electrode, $\mathrm{Ag} / \mathrm{AgCl}$ pseudoreference electrode; $100 \mathrm{mV} / \mathrm{s}$ scan rate; referenced to internal ferrocene standard. 

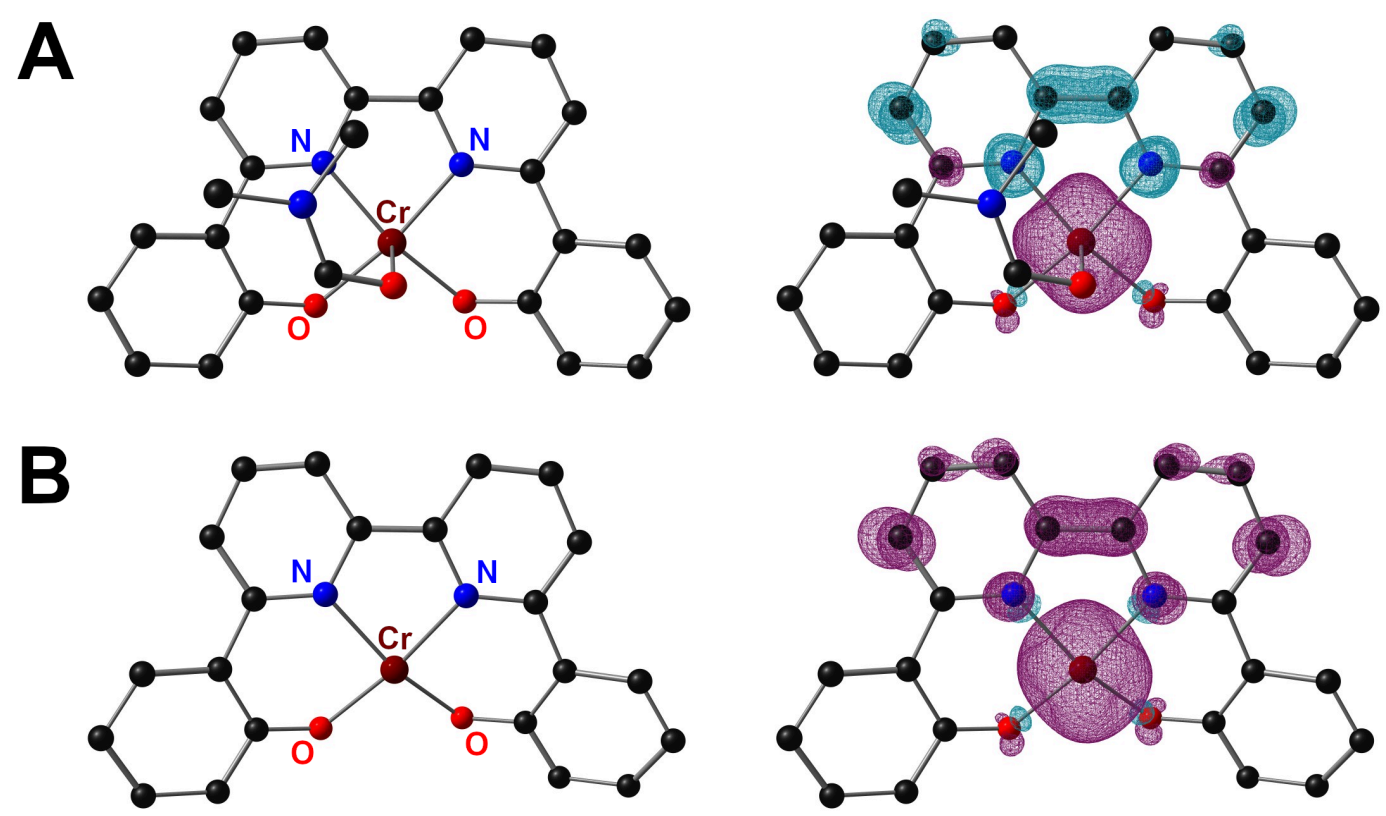

Figure S9. (A) Spin density plot of ${ }_{1}^{4} \mathbf{C r}^{-1}$, (B) spin density plot of ${ }_{0}^{6} \mathbf{C r}^{-1}$. H atoms and tBu groups omitted for clarity.

\section{$\mathrm{CO}_{2}$ Binding at $\mathrm{Cr}$}

Table S8. Relative potential (E) and corrected (concentration and quasiharmonic) triple zeta free energies ([qG]) for the transition states for $\mathrm{CO}_{2}$ binding at $\mathrm{Cr}$ from ${ }_{0}^{4} \mathrm{Cr}^{-1}$, in $\mathrm{kcal} / \mathrm{mol}$. No TSs were located in the sextet spin state.

\begin{tabular}{|c|c|c|c|}
\hline Species & $\mathbf{E}$ & {$[\mathbf{q G}]$} & $<\mathbf{S}^{\mathbf{}}>(\mathbf{D Z} / \mathbf{T Z})$ \\
\hline${ }_{0}^{4} \mathbf{T S 3}^{-1}+\mathrm{DMF}$ & -0.1 & +10.0 & $3.7636 / 3.7641$ \\
\hline${ }_{1}^{4} \mathbf{T S 3}^{-1}$ & -8.3 & +12.9 & $3.7647 / 3.7653$ \\
\hline
\end{tabular}

Table S9. Relative potential (E) and corrected (concentration and quasiharmonic) triple zeta free energies $([\mathrm{qG}])$ for $\left[\mathrm{Cr}(\mathrm{L})\left(\mathrm{CO}_{2}\right)(\mathrm{DMF})_{\mathrm{n}}\right]^{-}(\mathrm{n}=0,1)$ species, in $\mathrm{kcal} / \mathrm{mol}$. Optimizations to obtain ${ }_{0}^{6} \mathbf{C r}\left(\mathbf{C O}_{2}\right){ }^{-1}$ resulted in spontaneous release of $\mathrm{CO}_{2}$.

\begin{tabular}{|c|c|c|c|}
\hline Species & $\mathbf{E}$ & {$[\mathbf{q G}]$} & $<\mathbf{S}^{2}>(\mathbf{D Z} / \mathbf{T Z})$ \\
\hline${ }_{0}^{4} \mathbf{C r}\left(\mathbf{C O}_{2}\right)^{-1}+\mathrm{DMF}$ & -7.3 & +4.7 & $3.7522 / 3.7522$ \\
\hline${ }_{1}^{4} \mathbf{C r}\left(\mathbf{C O}_{2}\right)^{-1}$ & -14.7 & +8.8 & $3.7512 / 3.7512$ \\
\hline${ }_{1}^{6} \mathbf{C r}\left(\mathbf{C O}_{2}\right)^{-1}$ & -1.2 & +20.2 & $8.7502 / 8.7502$ \\
\hline
\end{tabular}




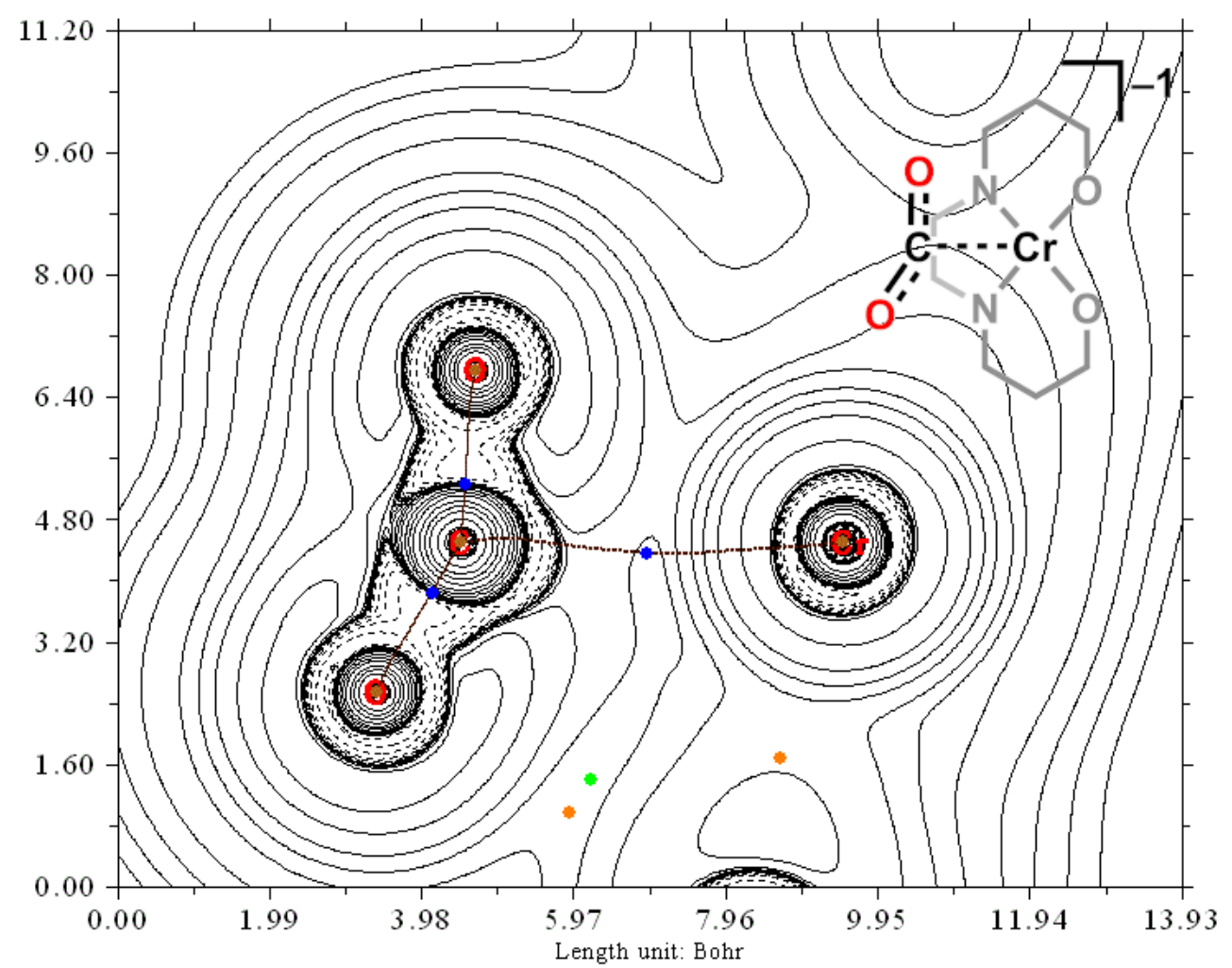

Figure S10. Laplacian of the electron density, $\nabla^{2} \rho$, plots of the $\mathrm{TS}$ for $\mathrm{CO}_{2}$ binding $\left({ }_{0}^{4} \mathbf{T S 3}^{-1}\right)$, showing a bcp between $\mathrm{Cr}$ and $\mathrm{C}$ and no bcps between $\mathrm{Cr}$ and $\mathrm{O}$ atoms.

A
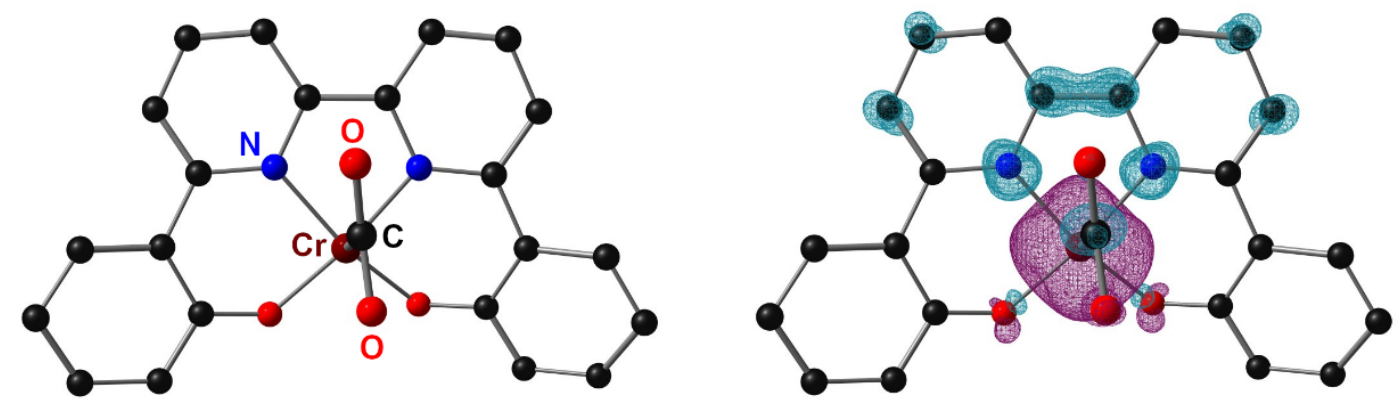

B
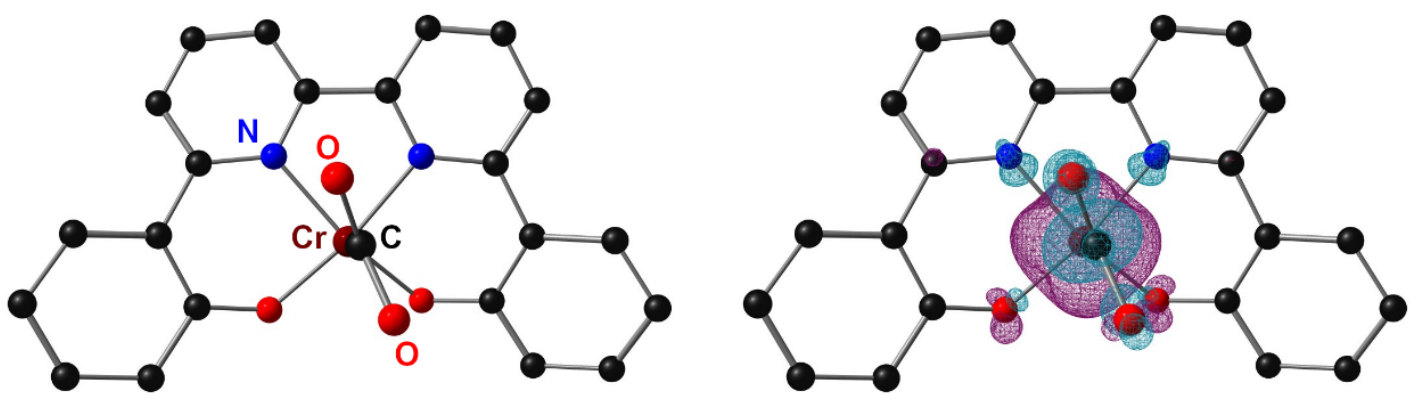

Figure S11. (A) Spin density plot of ${ }_{0}^{4} \mathbf{T S 3}^{-1}$, (B) spin density plot of ${ }_{0}^{4} \mathrm{Cr}\left(\mathrm{CO}_{2}\right)^{-1}$. H atoms and tBu groups omitted for clarity. 
A
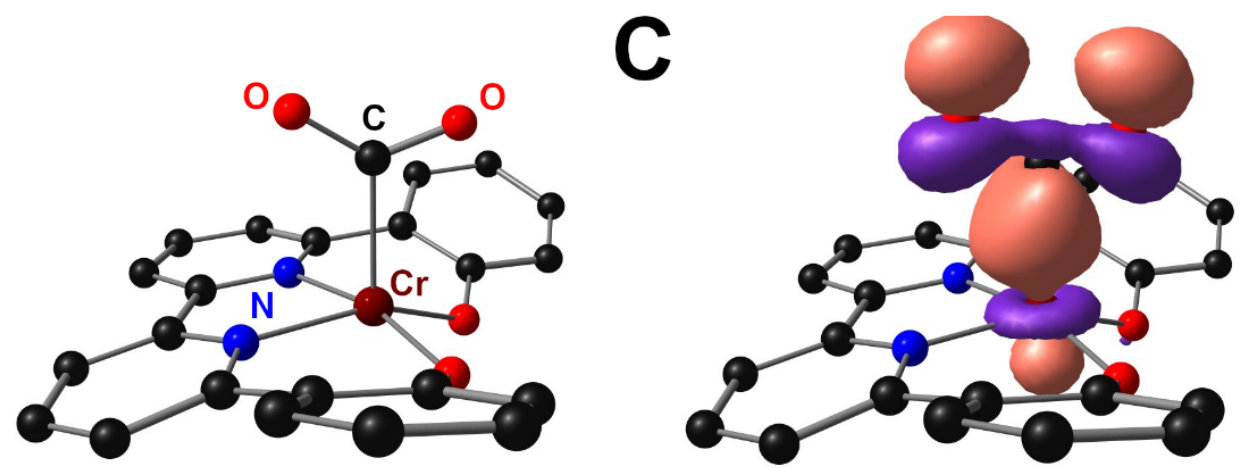

B
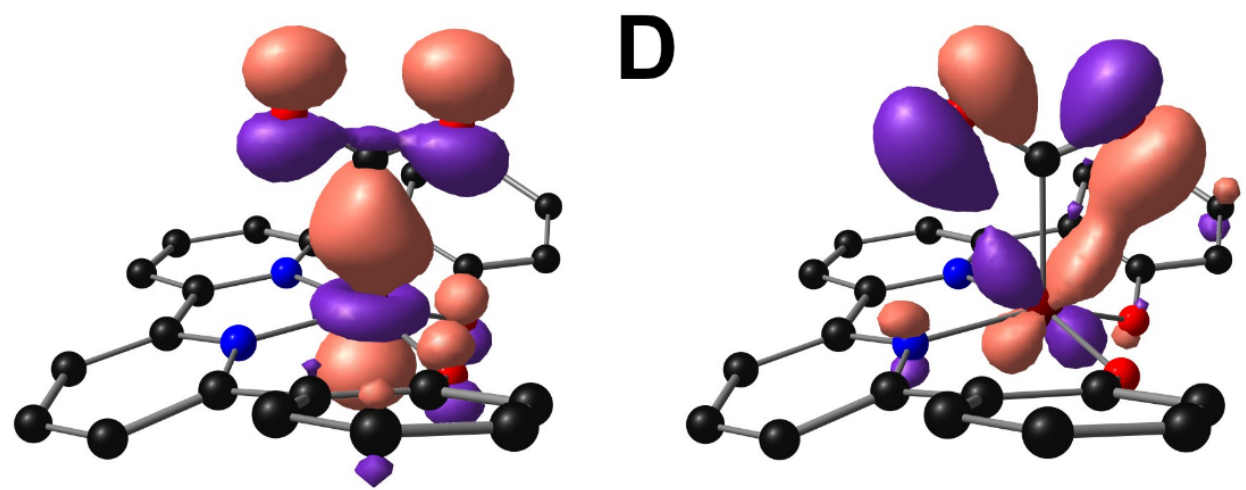

Figure S12. Molecular geometry (A), Highest SOMO (B), SOMO-1 (C) and SOMO-10 (D) of ${ }_{0}^{4} \mathbf{C r}\left(\mathrm{CO}_{2}\right)^{-1}$. H atoms and tBu groups omitted for clarity.

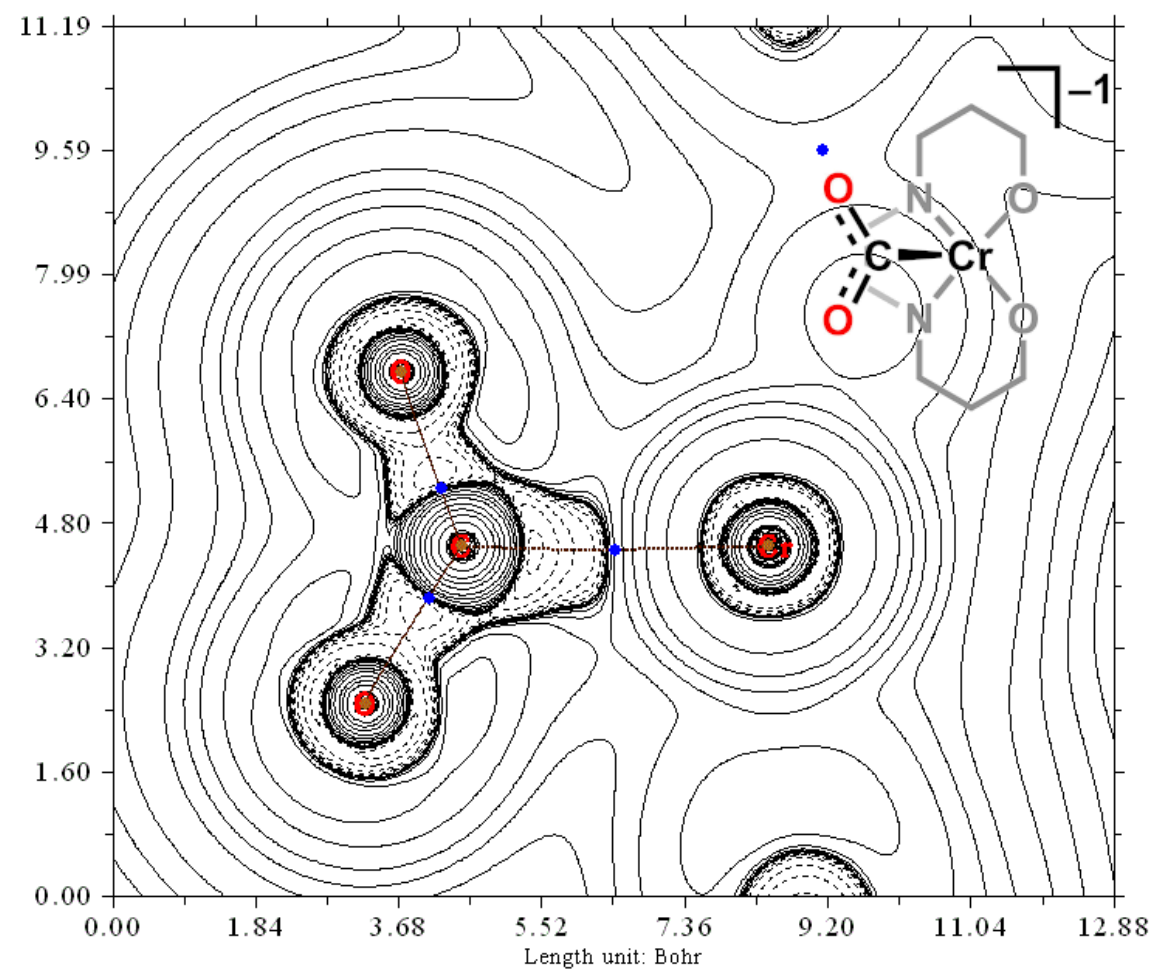

Figure S13. Laplacian of the electron density, $\nabla^{2} \rho$, plot of ${ }_{0}^{4} \mathbf{C r}\left(\mathbf{C O}_{2}\right)^{-1}$, showing a bcp only between $\mathrm{Cr}$ and $\mathrm{C}$. 


\section{$\mathrm{CO}_{2}$ binding at the bpy backbone}

While relaxed Potential Energy Surface scans revealed that $\mathrm{CO}_{2}$ binding at the 1 and 2 positions of the bpy backbone is not feasible, transition states could be located for the formation of $\mathrm{C}-\mathrm{C}$ bonds at the 3,4 and 5 positions of the bipyridine framework in the quartet and sextet surfaces, with and without explicit DMF. A complete study is only reported for positions 5 (TS12) and 3 (TS13), similar values were obtained for $\mathrm{CO}_{2}$ binding at the 4-position. All these barriers were at least $6.5 \mathrm{kcal} / \mathrm{mol}$ higher than the $\mathrm{Cr}-\mathrm{CO}_{2}$ bond formation one (Table S10), and the overall reactions were found to be highly endergonic, yielding products that were at least $10.8 \mathrm{kcal} / \mathrm{mol}$ less stable than the $\mathrm{Cr}-\mathrm{CO}_{2}$ adduct (Table S11). Based on these results, $\mathrm{CO}_{2}$ binding at the bpy backbone can be ruled out as a viable competitive pathway.

Table S10. Relative potential (E) and corrected (concentration and quasiharmonic) triple zeta free energies $([\mathrm{qG}])$ for the transition states for $\mathrm{CO}_{2}$ binding at the bpy (positions 5 and 3) from ${ }_{0}^{4} \mathbf{C r}^{-1}$, in kcal $/ \mathrm{mol}$.

\begin{tabular}{|c|c|c|c|}
\hline Species & $\mathbf{E}$ & [qG] & $<\mathrm{S}^{2}>(\mathrm{DZ} / \mathrm{TZ})$ \\
\hline${ }_{0}^{4} \mathrm{TS} 2^{-1}+\mathrm{DMF}$ & +6.4 & +17.2 & 3.7884 / 3.7886 \\
\hline${ }_{0}^{4} \mathrm{TS13}^{-1}+\mathrm{DMF}$ & +9.0 & +19.6 & $3.7872 / 3.7875$ \\
\hline${ }_{0}^{6} \mathrm{TS} 2^{-1}+\mathrm{DMF}$ & +6.6 & +16.7 & $8.7501 / 8.7501$ \\
\hline${ }_{0}^{6} \mathrm{TS13}^{-1}+\mathrm{DMF}$ & +8.6 & +18.5 & $8.7501 / 8.7501$ \\
\hline${ }_{1}^{4} \mathrm{TS}_{12}-1$ & -3.5 & +18.7 & 3.7889 / 3.7889 \\
\hline${ }_{1}^{4} \mathbf{T S 1 3}^{-1}$ & -0.9 & +22.2 & $3.7869 / 3.7871$ \\
\hline${ }_{1}^{6} \mathrm{TS}_{12}{ }^{-1}$ & -1.6 & +19.6 & $8.7501 / 8.7501$ \\
\hline${ }_{1}^{6} \mathbf{T S 1 3}^{-1}$ & +0.4 & +21.5 & $8.7501 / 8.7501$ \\
\hline
\end{tabular}


Table S11. Relative potential (E) and corrected (concentration and quasiharmonic) triple zeta free energies ([qG]) for the $\mathrm{CO}_{2}$ binding adducts at the bpy (positions 5 and 3 ) from ${ }_{0}^{4} \mathrm{Cr}^{-1}$, in $\mathrm{kcal} / \mathrm{mol}$.

\begin{tabular}{|c|c|c|c|}
\hline Species & $\mathbf{E}$ & [qG] & $<\mathrm{S}^{2}>(\mathrm{DZ} / \mathrm{TZ})$ \\
\hline${ }_{0}^{4} \mathrm{bpyCO}_{2}-5^{-1}+\mathrm{DMF}$ & +3.5 & +15.6 & $3.8015 / 3.8012$ \\
\hline${ }_{0}^{4} \mathbf{b p y C O}_{2}-3^{-1}+\mathrm{DMF}$ & +5.6 & +17.7 & 3.7984 / 3.7984 \\
\hline${ }_{0}^{6} \mathrm{bpyCO}_{2}-5^{-1}+\mathrm{DMF}$ & +3.1 & +15.1 & $8.7504 / 8.7504$ \\
\hline${ }_{0}^{6} \mathbf{b p y C O}_{2}-3^{-1}+\mathrm{DMF}$ & +4.8 & +16.8 & $8.7504 / 8.7504$ \\
\hline${ }_{1}^{4} \mathrm{bpyCO}_{2}-5^{-1}$ & -6.5 & +17.4 & $3.8020 / 3.8017$ \\
\hline${ }_{1}^{4} \mathrm{bpyCO}_{2-3}-1$ & -4.2 & +17.5 & $3.7980 / 3.7981$ \\
\hline${ }_{1}^{6} \mathrm{bpyCO}_{2}-5^{-1}$ & -5.5 & +17.7 & $8.7504 / 8.7504$ \\
\hline${ }_{1}^{6} \mathrm{bpyCO}_{2-3}-1$ & -5.1 & +18.1 & $8.7504 / 8.7504$ \\
\hline
\end{tabular}




\section{First Protonation}

Table S12. Potential (E) and corrected (concentration and quasiharmonic) triple zeta free energies $([\mathrm{qG}])$ for the $\mathrm{PhOH}$ adduct of ${ }_{1}^{4} \mathbf{C r}\left(\mathbf{C O}_{2}\right)^{-1}$ and the following protonation transition state $\left({ }_{1}^{4} \mathbf{T S} 4^{-1}\right)$ relative to ${ }_{1}^{4} \mathbf{C r}\left(\mathbf{C O}_{2}\right){ }^{-1}$ and independently computed $\mathrm{PhOH}$ and DMF, in $\mathrm{kcal} / \mathrm{mol}$.

\begin{tabular}{|c|c|c|c|}
\hline Species & $\mathbf{E}$ & {$[\mathbf{q G}]$} & $<\mathbf{S}^{\mathbf{2}}>\mathbf{( \mathbf { D Z } / \mathbf { T Z } )}$ \\
\hline${ }_{1}^{4} \mathbf{C r}\left(\mathbf{C O} \mathbf{O}_{2}{ }^{-1} \cdot \mathrm{PhOH}\right.$ & -26.8 & -1.4 & $3.7507 / 3.7507$ \\
\hline${ }_{1}^{4} \mathbf{T S 4}^{-1}$ & -25.4 & -2.0 & $3.7505 / 3.7505$ \\
\hline
\end{tabular}

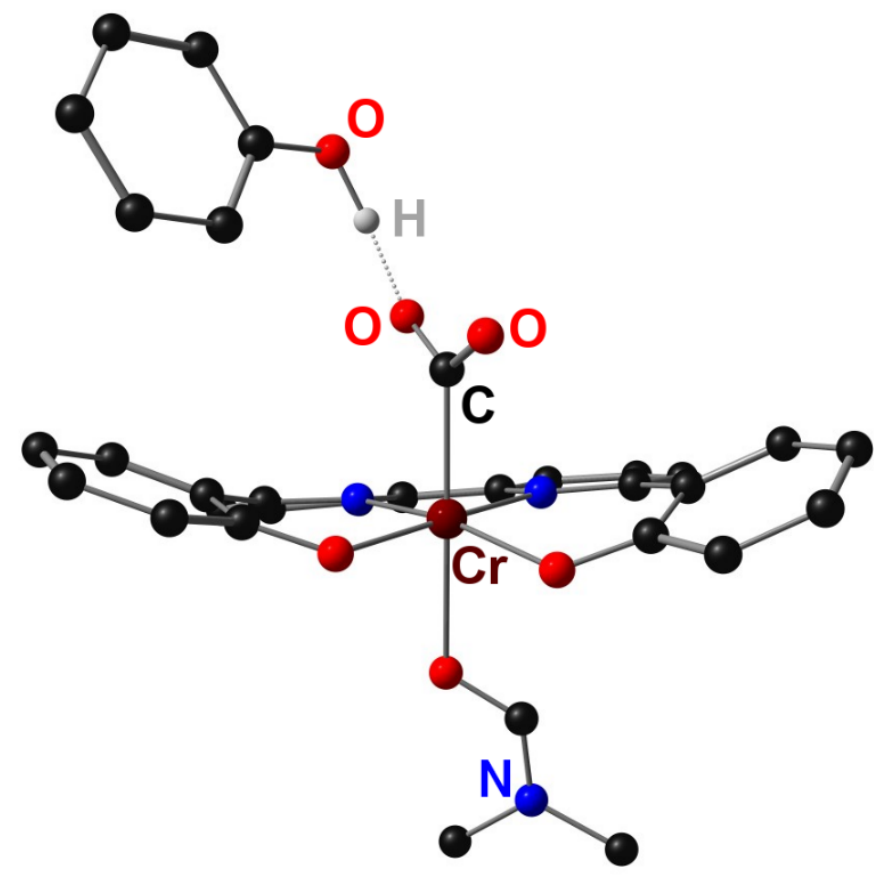

Figure S14. Molecular geometry of ${ }_{1}^{4} \mathrm{Cr}\left(\mathrm{CO}_{2}\right)^{-1} \cdot \mathrm{PhOH}$. For clarity, tBu groups and most $\mathrm{H}$ atoms have been omitted. 

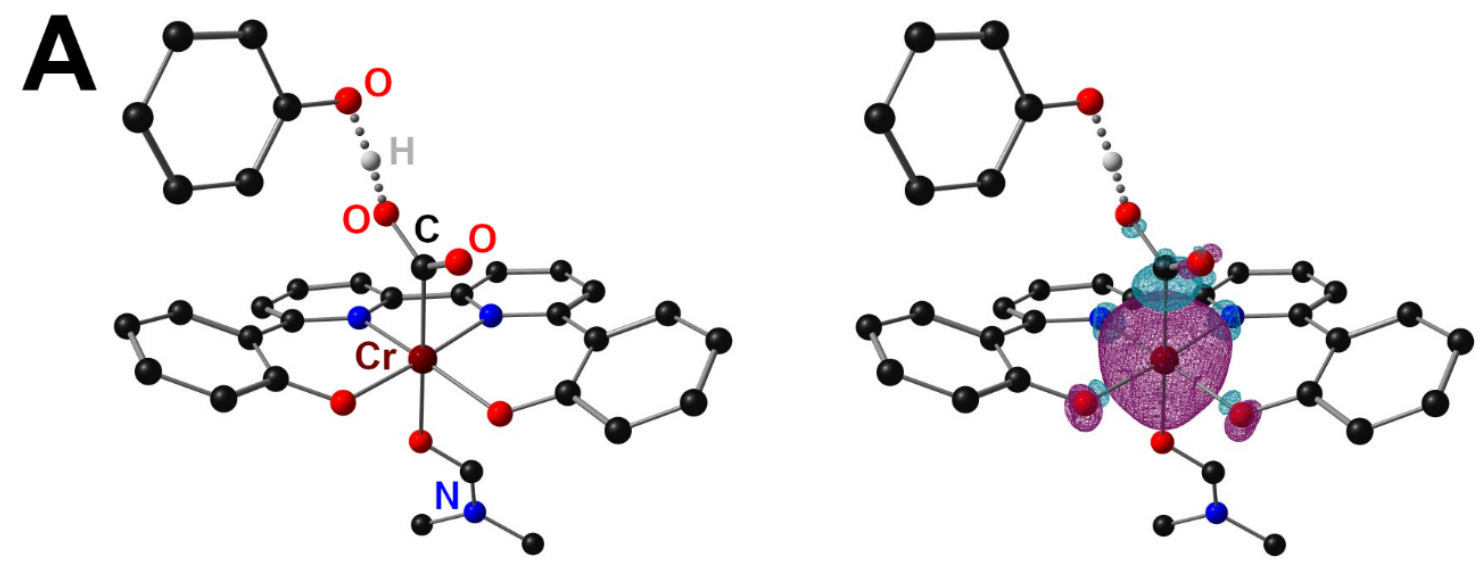

B
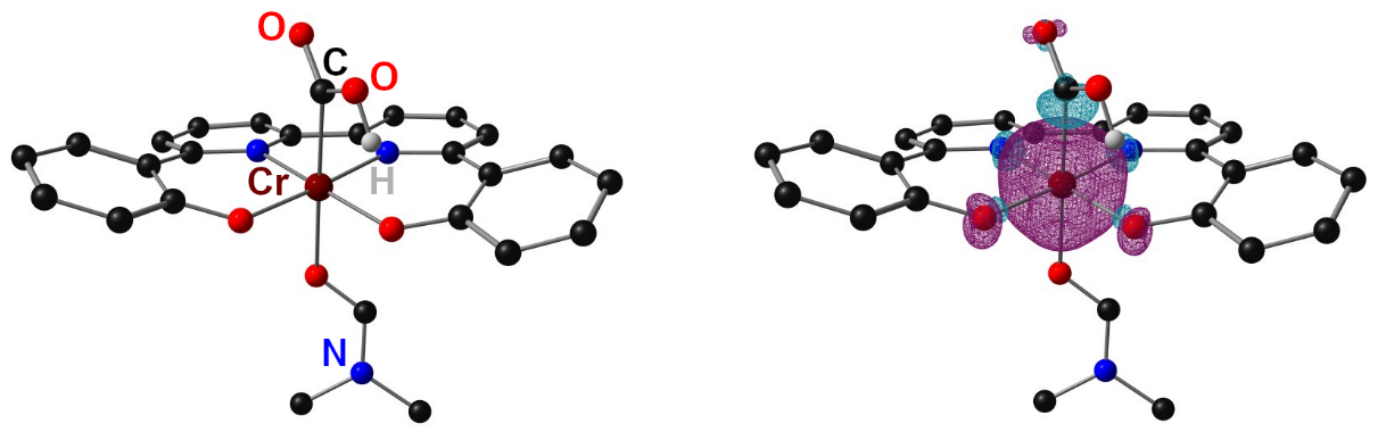

Figure S15. (A) Spin density plot of ${ }_{1}^{4} \mathrm{TS}^{-1}$, (B) spin density plot of ${ }_{1}^{4} \mathrm{Cr}\left(\mathrm{CO}_{2} \mathbf{H}\right)^{0}$. For clarity, $\mathrm{tBu}$ groups and most $\mathrm{H}$ atoms have been omitted.

Table S13. Relative potential (E) and corrected (concentration and quasiharmonic) triple zeta free energies $([\mathrm{qG}])$ for the metal-bound hydroxycarbonyl $\mathrm{Cr}(\mathrm{L})\left(\mathrm{CO}_{2} \mathrm{H}\right)(\mathrm{DMF})_{\mathrm{n}}(\mathrm{n}$ $=0,1)$, in $\mathrm{kcal} / \mathrm{mol}$.

\begin{tabular}{|c|c|c|c|}
\hline Species & $\mathbf{E}$ & [qG] & $<\mathrm{S}^{2}>(\mathrm{DZ} / \mathrm{TZ})$ \\
\hline${ }_{0}^{2} \mathrm{Cr}\left(\mathrm{CO}_{2} \mathbf{H}\right){ }^{0}+\mathrm{DMF}$ & +36.8 & +25.1 & $0.7889 / 0.7892$ \\
\hline${ }_{0}^{4} \mathrm{Cr}\left(\mathrm{CO}_{2} \mathbf{H}\right)^{0}+\mathrm{DMF}$ & +15.9 & +3.8 & $3.7505 / 3.7505$ \\
\hline${ }_{0}^{6} \mathrm{Cr}\left(\mathrm{CO}_{2} \mathrm{H}\right){ }^{0}+\mathrm{DMF}$ & +65.5 & +50.5 & $8.7512 / 8.7512$ \\
\hline${ }_{1}^{2} \mathrm{Cr}\left(\mathrm{CO}_{2} \mathrm{H}\right){ }^{0}$ & +21.3 & +22.0 & $0.7976 / 0.7986$ \\
\hline${ }_{1}^{4} \mathrm{Cr}\left(\mathrm{CO}_{2} \mathrm{H}\right){ }^{0}$ & 0.0 & +0.0 & $3.7503 / 3.7503$ \\
\hline${ }_{1}^{6} \mathrm{Cr}\left(\mathrm{CO}_{2} \mathrm{H}\right){ }^{0}$ & +49.0 & +46.8 & $8.7510 / 8.7510$ \\
\hline
\end{tabular}




$$
\left[\mathrm{Cr}(\mathrm{L})\left(\mathrm{CO}_{2} \mathrm{H}\right)(\mathrm{DMF})\right]+\mathrm{PhOH} \rightleftharpoons[\mathrm{Cr}(\mathrm{L})(\mathrm{CO})(\mathrm{DMF})]^{+}+\mathrm{H}_{2} \mathrm{O}+\mathrm{PhO}^{-}
$$

Eq S5: $\Delta[\mathrm{qG}]=10.2 \mathrm{kcal} / \mathrm{mol}$

$$
\left[\mathrm{Cr}(\mathrm{L})\left(\mathrm{CO}_{2} \mathrm{H}\right)(\mathrm{DMF})\right]+2 \mathrm{PhOH} \rightleftharpoons[\mathrm{Cr}(\mathrm{L})(\mathrm{CO})(\mathrm{DMF})]^{+}+\mathrm{H}_{2} \mathrm{O}+\left[(\mathrm{PhO})_{2} \mathrm{H}\right]^{-}
$$

Eq S6: $\Delta[\mathrm{qG}]=5.1 \mathrm{kcal} / \mathrm{mol}$

Table S14. Relative potential (E) and corrected (concentration and quasiharmonic) triple zeta free energies $([\mathrm{qG}])$ for the cationic carbonyl complex $[\mathrm{Cr}(\mathrm{L})(\mathrm{CO})(\mathrm{DMF})]^{+}$, in

\begin{tabular}{|c|c|c|c|}
\hline Species & $\mathbf{E}$ & {$[\mathbf{q G}]$} & $<\mathrm{S}^{2}>(\mathrm{DZ} / \mathrm{TZ})$ \\
\hline${ }_{1}^{2} \mathrm{Cr}(\mathrm{CO}){ }^{+1}$ & +15.3 & +15.9 & $0.7774 / 0.7778$ \\
\hline${ }_{0}^{4} \mathrm{Cr}(\mathbf{C O}){ }^{+1}$ & +25.9 & +13.1 & $3.7505 / 3.7505$ \\
\hline${ }_{1}^{4} \mathrm{Cr}(\mathrm{CO}){ }^{+1}$ & 0.0 & 0.0 & $3.7503 / 3.7503$ \\
\hline
\end{tabular}
$\mathrm{kcal} / \mathrm{mol}$. 


\section{Reduction}

Table S15. Relative potential (E) and corrected (concentration and quasiharmonic) triple zeta free energies ([qG]) for the reduced metal-bound hydroxycarbonyl $\left[\mathrm{Cr}(\mathrm{L})\left(\mathrm{CO}_{2} \mathrm{H}\right)(\mathrm{DMF})_{\mathrm{n}}\right]^{-}(\mathrm{n}=0,1)$, in $\mathrm{kcal} / \mathrm{mol}$. Entries in red presented large spin contamination.

\begin{tabular}{|c|c|c|c|}
\hline Species & $\mathbf{E}$ & {$[q G]$} & $<\mathrm{S}^{2}>(\mathrm{DZ} / \mathrm{TZ})$ \\
\hline${ }_{0}^{1} \mathrm{Cr}\left(\mathrm{CO}_{2} \mathbf{H}\right)^{-1}+\mathrm{DMF}$ & +36.6 & +26.4 & $2.6078 / 2.7099$ \\
\hline${ }_{0}^{3} \mathrm{Cr}\left(\mathrm{CO}_{2} \mathbf{H}\right)^{-1}+\mathrm{DMF}$ & +12.1 & +0.6 & $2.0557 / 0.0568$ \\
\hline${ }_{0}^{5} \mathrm{Cr}\left(\mathrm{CO}_{2} \mathbf{H}\right)^{-1}+\mathrm{DMF}$ & +17.2 & +5.0 & $6.0005 / 6.0005$ \\
\hline${ }_{1}^{1} \mathrm{Cr}\left(\mathrm{CO}_{2} \mathrm{H}\right)^{-1}$ & +21.9 & +23.9 & $2.7663 / 2.8533$ \\
\hline${ }_{1}^{3} \mathrm{Cr}\left(\mathrm{CO}_{2} \mathrm{H}\right)^{-1}$ & 0.0 & +0.0 & $2.0527 / 2.0535$ \\
\hline${ }_{1}^{5} \mathrm{Cr}\left(\mathrm{CO}_{2} \mathrm{H}\right)^{-1}$ & +4.9 & +4.4 & $6.0004 / 6.0004$ \\
\hline
\end{tabular}

$$
P H N Z^{-}+{ }_{1}^{4} \operatorname{Cr}\left(\mathrm{CO}_{2} \mathrm{H}\right){ }^{0} \rightleftharpoons P H N Z+{ }_{1}^{3} \mathrm{Cr}\left(\mathrm{CO}_{2} \mathrm{H}\right)^{-1}
$$

Eq S7: $\Delta[\mathrm{qG}]=9.2 \mathrm{kcal} / \mathrm{mol}$

$\mathrm{V}$ vs $\mathrm{PHNZ}^{-} / \mathrm{PHNZ}=-0.40 . \mathrm{V}$ vs $\mathrm{Fc}^{+} / \mathrm{Fc}=-2.03$. Calibrated $\mathrm{V}$ vs $\mathrm{Fc}^{+} / \mathrm{Fc}=-1.92$.

A
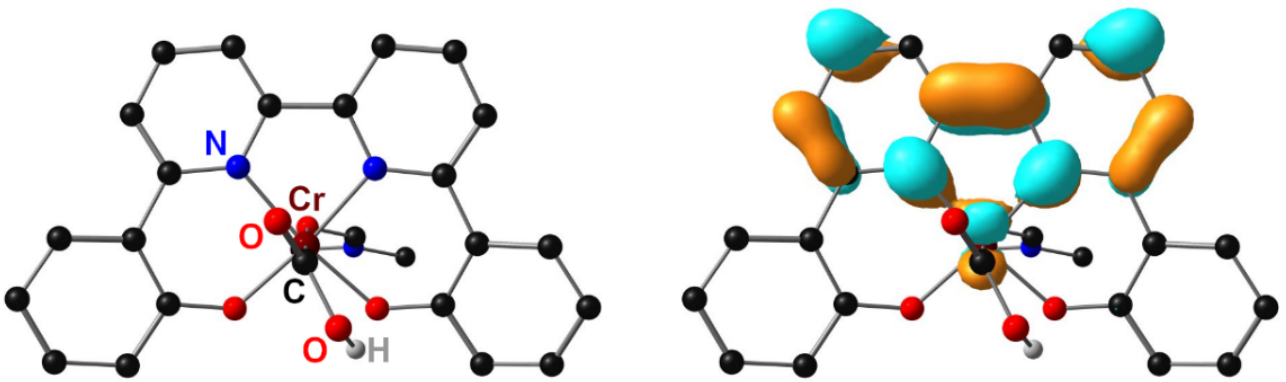

B
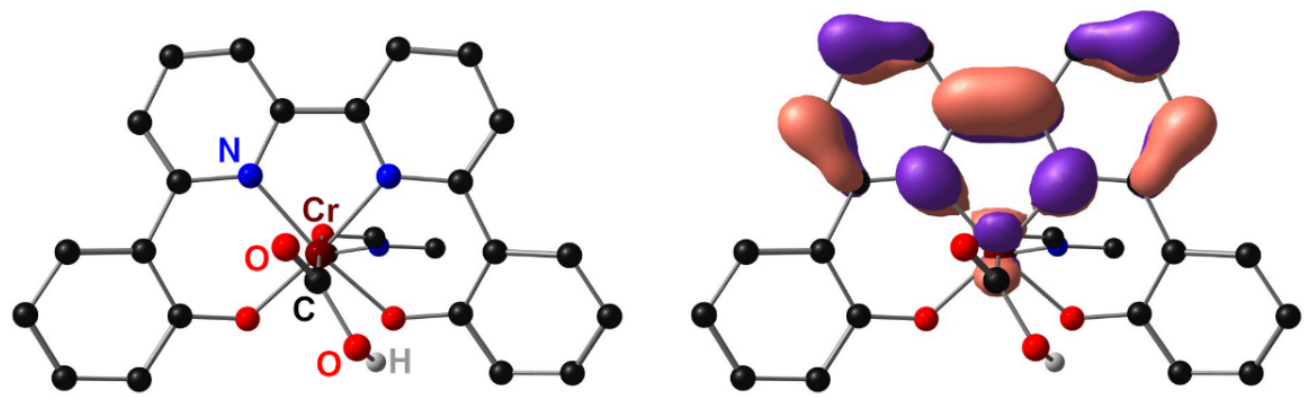

Figure S16. (A) LUMO of ${ }_{1}^{4} \mathrm{Cr}\left(\mathrm{CO}_{2} \mathrm{H}\right){ }^{0}$, (B) highest SOMO of ${ }_{1}^{3} \mathrm{Cr}\left(\mathrm{CO}_{2} \mathrm{H}\right)^{-1}$. For clarity, $\mathrm{tBu}$ groups and most $\mathrm{H}$ atoms have been omitted. 
A
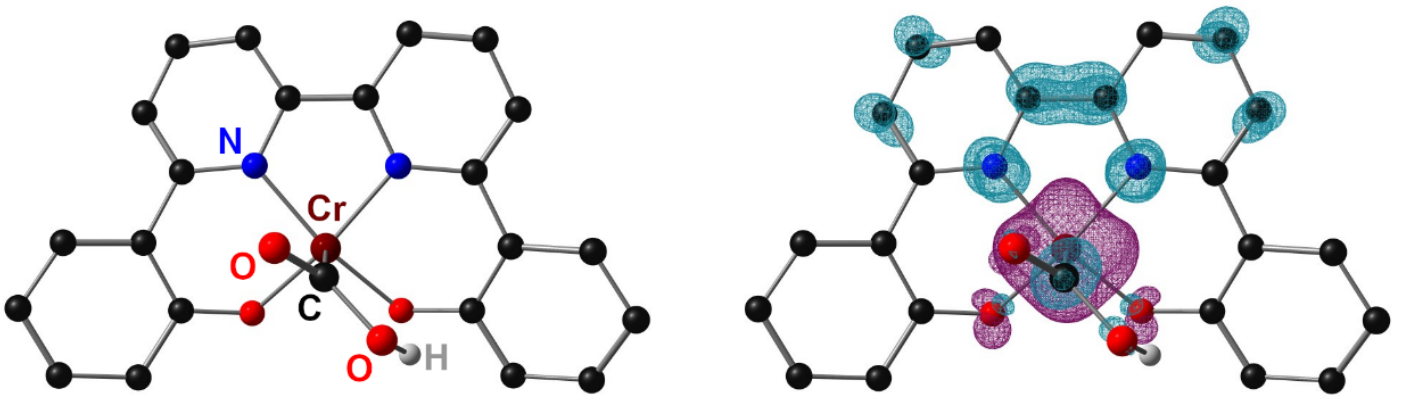

B
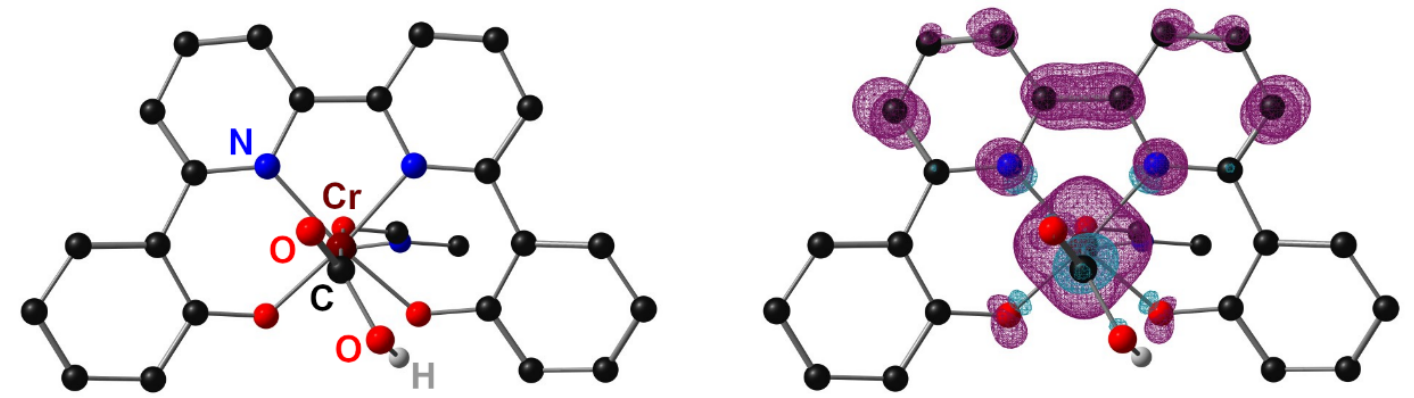

?
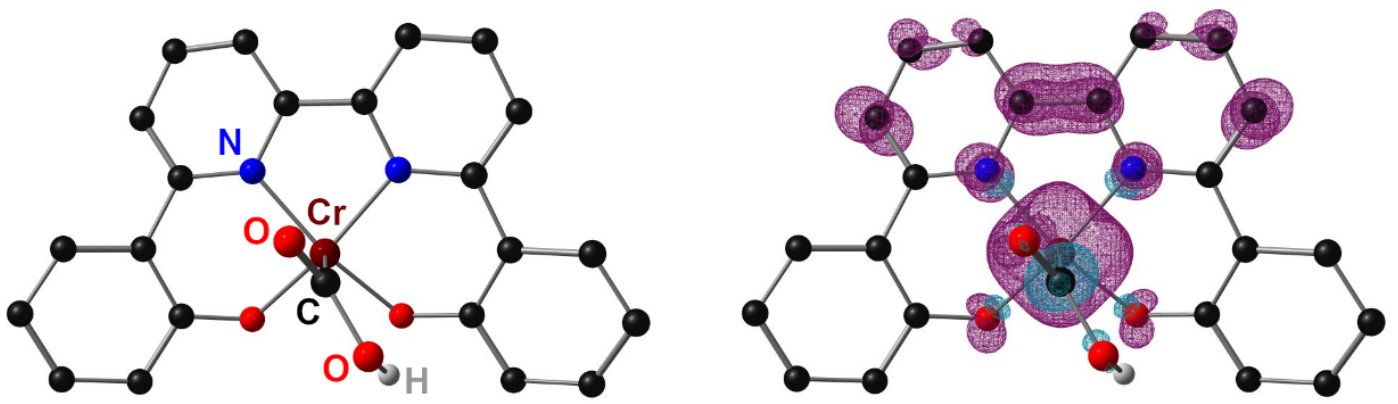

Figure S17. Spin density plot of (A) ${ }_{0}^{3} \mathrm{Cr}\left(\mathrm{CO}_{2} \mathrm{H}\right){ }^{-1}$, (B) ${ }_{1}^{5} \mathrm{Cr}\left(\mathrm{CO}_{2} \mathrm{H}\right){ }^{-1}$ and (C) ${ }_{0}^{5} \mathrm{Cr}\left(\mathrm{CO}_{2} \mathrm{H}\right)^{-1}$. For clarity, tBu groups and most $\mathrm{H}$ atoms have been omitted. 
Table S16. Relative potential (E) and corrected (concentration and quasiharmonic) triple zeta free energies $([\mathrm{qG}])$ for the transition states for the protonation of the metal-bound hydroxycarbonyl ${ }_{1}^{3} \mathbf{C r}\left(\mathbf{C O}_{2} \mathbf{H}\right)^{-1}$, in kcal/mol.

\begin{tabular}{|c|c|c|c|}
\hline Species & $\mathbf{E}$ & [qG] & $<\mathrm{S}^{2}>(\mathrm{DZ} / \mathrm{TZ})$ \\
\hline${ }_{0}^{3} \mathrm{Cr}\left(\mathrm{CO}_{2} \mathrm{H}\right)^{-1} \cdot \mathrm{PhOH}+\mathrm{DMF}$ & +0.8 & +3.5 & $2.0429 / 2.0442$ \\
\hline${ }_{0}^{3} \mathbf{T S 5}^{-1}+\mathrm{DMF}$ & +15.4 & +14.0 & $2.0091 / 2.0098$ \\
\hline${ }_{0}^{5} \mathrm{Cr}\left(\mathrm{CO}_{2} \mathrm{H}\right)^{-1} \cdot \mathrm{PhOH}+\mathrm{DMF}$ & +4.5 & +6.4 & $6.0005 / 6.0005$ \\
\hline${ }_{0}^{5} \mathrm{TS5}^{-1}+\mathrm{DMF}$ & +12.9 & +11.1 & $6.0001 / 6.0001$ \\
\hline${ }_{1}^{3} \mathrm{Cr}\left(\mathrm{CO}_{2} \mathrm{H}\right)^{-1} \cdot \mathrm{PhOH}$ & -13.8 & +0.6 & $2.0455 / 2.0464$ \\
\hline${ }_{1}^{3} \mathrm{TS5}^{-1}$ & -1.2 & +10.5 & $2.0193 / 2.0204$ \\
\hline${ }_{1}^{5} \mathrm{Cr}\left(\mathrm{CO}_{2} \mathrm{H}\right)^{-1} \cdot \mathrm{PhOH}$ & -8.9 & +5.5 & 6.0004 / 6.0004 \\
\hline${ }_{1}^{5} \mathbf{T S 5}^{-1}$ & +6.9 & +18.6 & $6.0003 / 6.0003$ \\
\hline
\end{tabular}



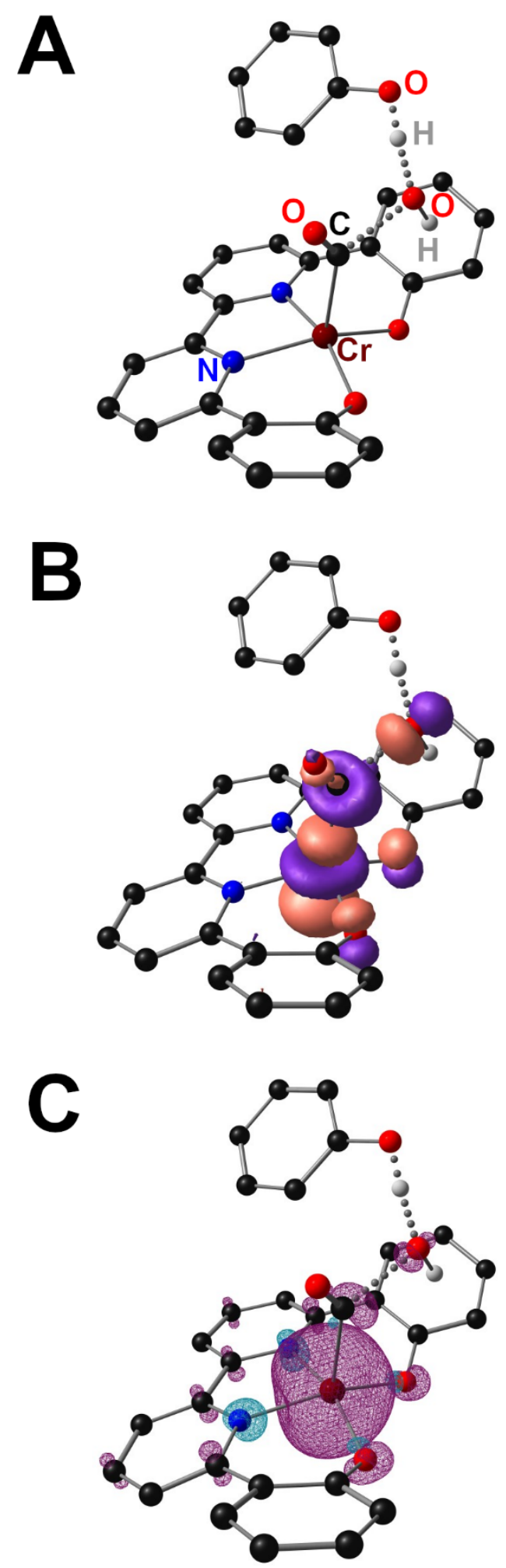

Figure S18. (A) Molecular geometry, (B) highest SOMO and (C) spin density plot of ${ }_{0}^{5} \mathbf{T S 5}^{-1}$. For clarity, tBu groups and most $\mathrm{H}$ atoms have been omitted. 


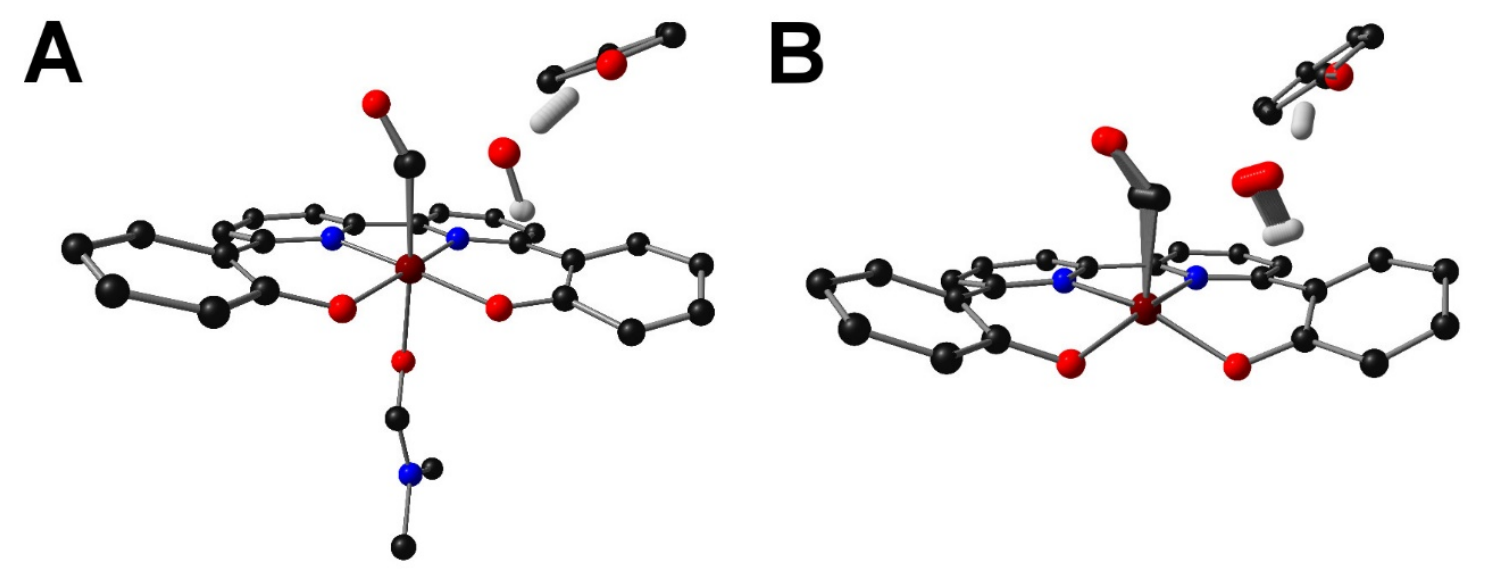

Figure S19. Overlapped displaced geometries along the imaginary frequency for ${ }_{1}^{3} \mathbf{T S 5}^{-1}$ (A) and ${ }_{0}^{5} \mathbf{T S 5}^{-1}$ (B). Scale displacement \pm 0.35 . For clarity, tBu groups and most $\mathrm{H}$ atoms have been omitted. 


\section{Second Protonation: Dianionic Pathway}

Table S17. Relative potential (E) and corrected (concentration and quasiharmonic) triple zeta free energies $([\mathrm{qG}])$ for the doubly reduced metal-bound hydroxycarbonyl $\left[\mathrm{Cr}(\mathrm{L})\left(\mathrm{CO}_{2} \mathrm{H}\right)(\mathrm{DMF})_{\mathrm{n}}\right]^{-2}(\mathrm{n}=0,1)$ and TSs for their protonation, in kcal/mol. ${ }_{1}^{6} \mathbf{T S 5}^{-2}$ could not be found, but relaxed potential energy surface scans suggest it is not the lowest energy for the dianionic pathway. Entries in red presented large spin contamination.

\begin{tabular}{|c|c|c|c|}
\hline Species & $\mathbf{E}$ & [qG] & $<\mathrm{S}^{2}>(\mathrm{DZ} / \mathrm{TZ})$ \\
\hline${ }_{0}^{2} \mathrm{Cr}\left(\mathrm{CO}_{2} \mathbf{H}\right)^{-2}+\mathrm{DMF}$ & +6.7 & +6.3 & $2.5929 / 2.6617$ \\
\hline${ }_{0}^{4} \mathrm{Cr}\left(\mathrm{CO}_{2} \mathbf{H}\right)^{-2}+\mathrm{DMF}$ & 0.0 & 0.0 & $3.7532 / 3.7532$ \\
\hline${ }_{0}^{6} \mathrm{Cr}\left(\mathrm{CO}_{2} \mathbf{H}\right)^{-2}+\mathrm{DMF}$ & +15.8 & +14.2 & $8.7507 / 8.7507$ \\
\hline${ }_{1}^{2} \mathrm{Cr}\left(\mathrm{CO}_{2} \mathrm{H}\right)^{-2}$ & -4.4 & +7.2 & $3.0591 / 3.0995$ \\
\hline${ }_{1}^{4} \mathrm{Cr}\left(\mathrm{CO}_{2} \mathrm{H}\right){ }^{-2}$ & -7.2 & +3.9 & $3.7586 / 3.7585$ \\
\hline${ }_{1}^{6} \mathrm{Cr}\left(\mathrm{CO}_{2} \mathrm{H}\right){ }^{-2}$ & +4.0 & +14.6 & $8.7506 / 8.7506$ \\
\hline${ }_{0}^{2} \mathbf{T S 5}^{-2}$ & +8.5 & +20.6 & $1.3582 / 1.3927$ \\
\hline${ }_{0}^{4} \mathrm{Cr}\left(\mathrm{CO}_{2} \mathrm{H}\right)^{-2} \cdot \mathrm{PhOH}$ & -14.3 & -3.2 & $3.7531 / 3.7531$ \\
\hline${ }_{0}^{4} \mathrm{TS5}^{-2}$ & -9.8 & +1.1 & $3.7747 / 3.7750$ \\
\hline${ }_{0}^{6}$ TS5 $^{-2}$ & -4.3 & +6.2 & $8.7501 / 8.7501$ \\
\hline${ }_{1}^{2} \mathbf{T S 5}^{-2}$ & -9.1 & +13.5 & $1.6975 / 1.7503$ \\
\hline${ }_{1}^{4} \mathrm{Cr}\left(\mathrm{CO}_{2} \mathrm{H}\right)^{-2} \cdot \mathrm{PhOH}$ & -21.9 & +3.7 & $3.7573 / 3.7572$ \\
\hline${ }_{1}^{4} \mathrm{TS5}^{-2}$ & -12.5 & +9.5 & $3.7564 / 3.7566$ \\
\hline${ }_{1}^{6} \mathbf{T S 5}^{-2}$ & +3.4 & +26.0 & $8.7504 / 8.7504$ \\
\hline
\end{tabular}

$$
P H N Z^{-}+{ }_{1}^{3} \operatorname{Cr}\left(\mathrm{CO}_{2} \mathrm{H}\right)^{-1} \rightleftharpoons P H N Z+{ }_{0}^{4} \boldsymbol{C r}\left(\mathrm{CO}_{2} \mathrm{H}\right)^{-2}+D M F
$$

Eq S8: $\Delta[\mathrm{qG}]=25.0 \mathrm{kcal} / \mathrm{mol}$

$\mathrm{V}$ vs $\mathrm{PHNZ}^{-} / \mathrm{PHNZ}=-1.08 . \mathrm{V}$ vs Fc$/ \mathrm{Fc}=-2.71$. Calibrated $\mathrm{V}$ vs $\mathrm{Fc}^{+} / \mathrm{Fc}=-2.53$. 

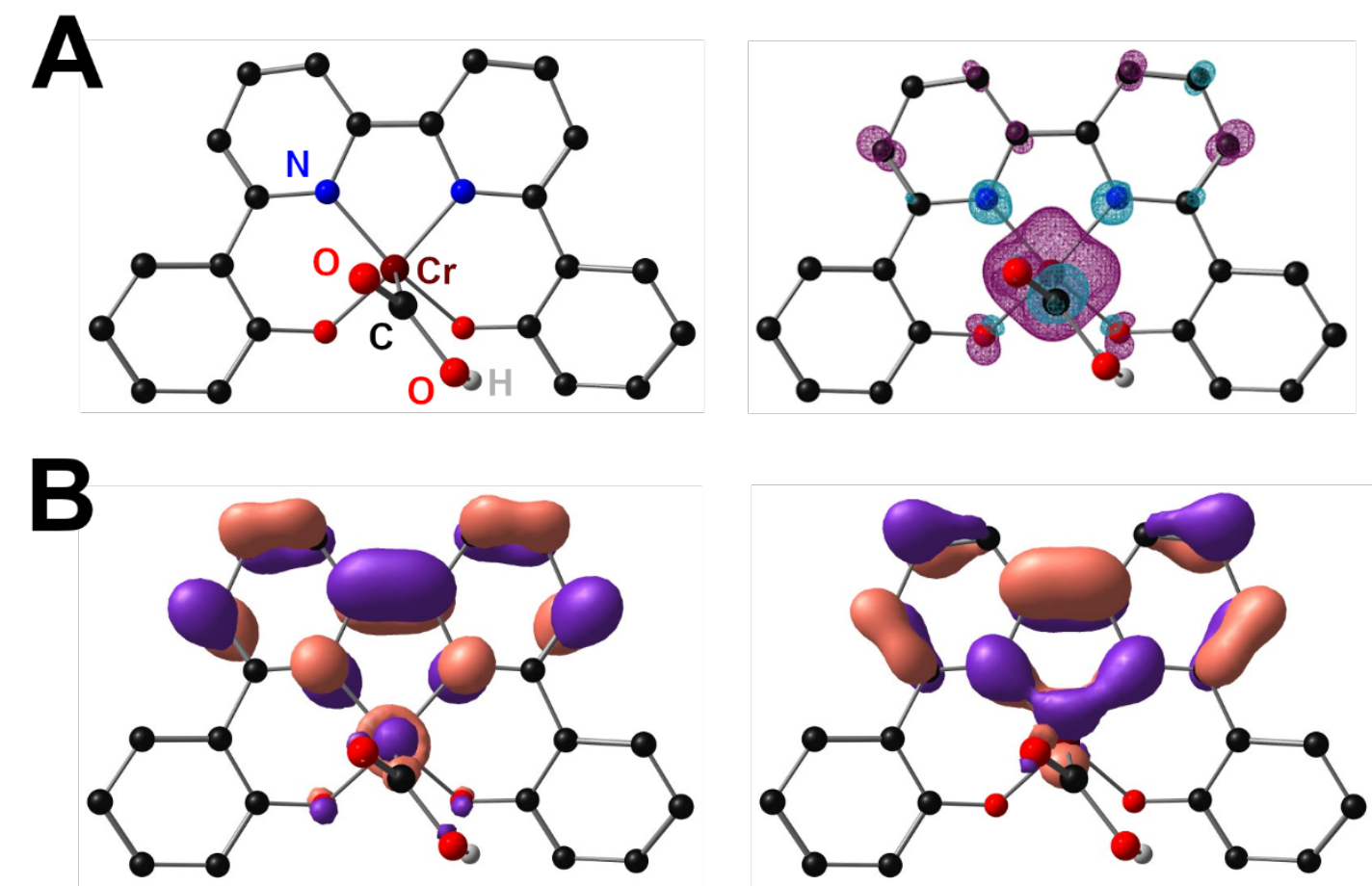

Figure S20. (A) Spin density and (B) highest SOMOs of ${ }_{0}^{4} \mathbf{C r}\left(\mathrm{CO}_{2} \mathbf{H}\right)^{-2}$, presenting opposite spin. For clarity, tBu groups and most $\mathrm{H}$ atoms have been omitted.
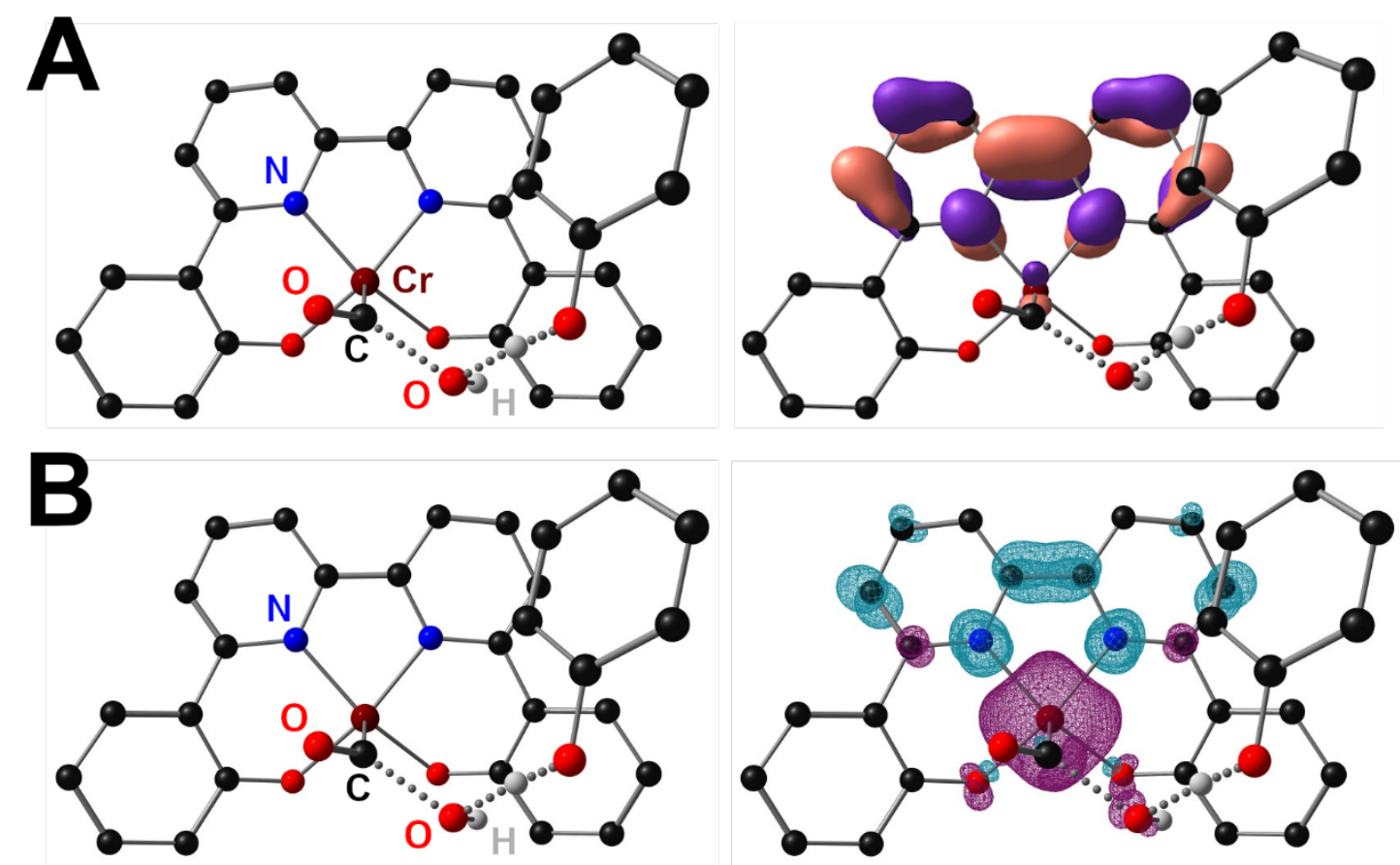

Figure S21. (A) Highest SOMO and (B) spin density of ${ }_{0}^{4} \mathbf{T S 5}^{-2}$. For clarity, tBu groups and most $\mathrm{H}$ atoms have been omitted. 


\section{Carbonyl Complexes}

Table S18. Relative potential (E) and corrected (concentration and quasiharmonic) triple zeta free energies $([\mathrm{qG}])$ for the neutral carbonyl complexes $\mathrm{Cr}(\mathrm{L})(\mathrm{CO})(\mathrm{DMF})_{\mathrm{n}}(\mathrm{n}=0$, $1)$, in $\mathrm{kcal} / \mathrm{mol}$.

\begin{tabular}{|c|c|c|c|}
\hline Species & $\mathbf{E}$ & {$[\mathbf{q G}]$} & $<\mathbf{S}^{2}>(\mathbf{D Z} / \mathbf{T Z})$ \\
\hline${ }_{0}^{1} \mathbf{C r}(\mathbf{C O})^{0}+\mathrm{DMF}$ & +26.7 & +15.9 & $0.0182 / 0.0189$ \\
\hline${ }_{0}^{3} \mathbf{C r}(\mathbf{C O})^{0}+\mathrm{DMF}$ & +16.8 & +4.6 & $2.0024 / 2.0025$ \\
\hline${ }_{0}^{5} \mathbf{C r}(\mathbf{C O})^{0}+\mathrm{DMF}$ & +15.3 & +0.6 & $6.0002 / 6.0002$ \\
\hline${ }_{1}^{1} \mathbf{C r}(\mathbf{C O})^{0}$ & +13.3 & +14.3 & $0.0272 / 0.0285$ \\
\hline${ }_{1}^{3} \mathbf{C r}(\mathbf{C O})^{0}$ & 0.0 & 0.0 & $2.0041 / 2.0043$ \\
\hline${ }^{5} \mathbf{C r}(\mathbf{C O})^{0}$ & +5.4 & +2.3 & $6.0002 / 6.0002$ \\
\hline
\end{tabular}

$$
\left[\mathrm{Cr}(\mathrm{L})\left(\mathrm{CO}_{2} \mathrm{H}\right)(\mathrm{DMF})\right]^{-}+\mathrm{PhOH} \rightleftharpoons[\mathrm{Cr}(\mathrm{L})(\mathrm{CO})(\mathrm{DMF})]+\mathrm{H}_{2} \mathrm{O}+\mathrm{PhO}^{-}
$$

Eq S9: $\Delta[\mathrm{qG}]=-9.2 \mathrm{kcal} / \mathrm{mol}$

$$
\begin{aligned}
& {\left[\mathrm{Cr}(\mathrm{L})\left(\mathrm{CO}_{2} \mathrm{H}\right)(\mathrm{DMF})\right]^{-}+2 \mathrm{PhOH} \rightleftharpoons[\mathrm{Cr}(\mathrm{L})(\mathrm{CO})(\mathrm{DMF})]+\mathrm{H}_{2} \mathrm{O}+\left[(\mathrm{PhO})_{2} \mathrm{H}\right]^{-}} \\
& \text {Eq S10: } \Delta[\mathrm{qG}]=-14.4 \mathrm{kcal} / \mathrm{mol}
\end{aligned}
$$

CO Release

$$
\begin{gathered}
{[C r(L)(C O)(D M F)]} \\
\quad \text { Eq S11: } \Delta[\mathrm{qG}]=-5.2 \mathrm{kcal} / \mathrm{mol}
\end{gathered}
$$




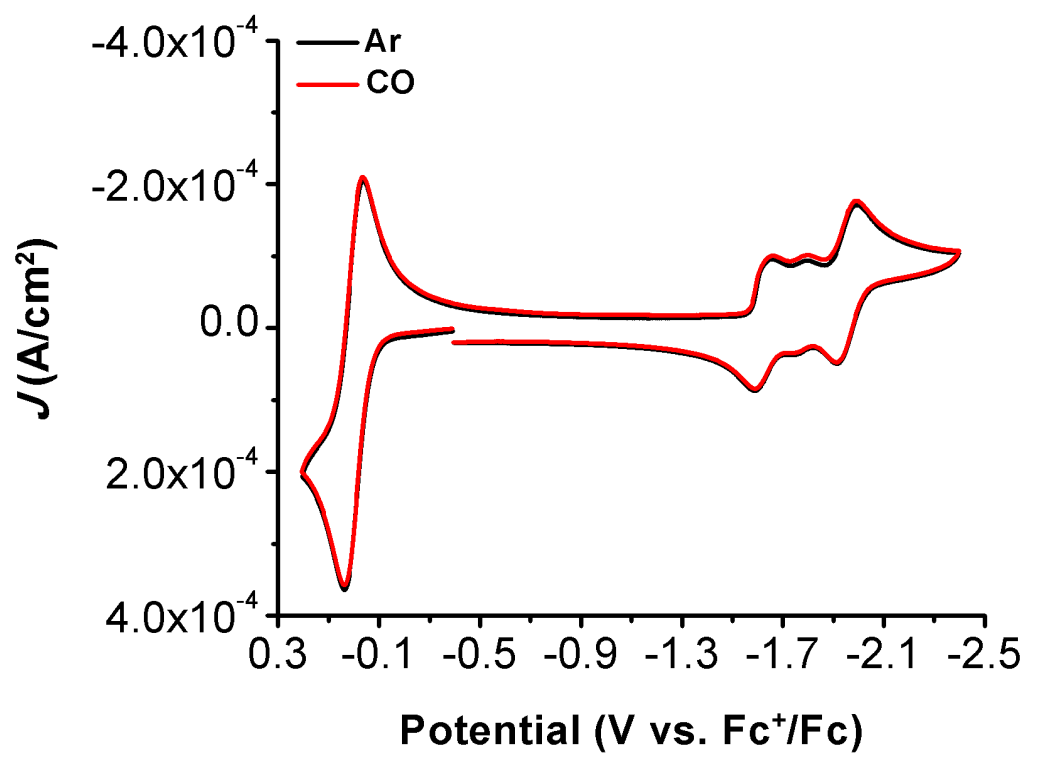

Figure S22. CVs of $1.0 \mathrm{mM} \mathrm{Cr}\left({ }^{\text {tbu }} \mathrm{dhbpy}\right) \mathrm{Cl}\left(\mathrm{H}_{2} \mathrm{O}\right)$ under $\mathrm{Ar}$ (black) and $\mathrm{CO}$ (red) saturation conditions. Conditions: $0.1 \mathrm{M} \mathrm{TBAPF} / \mathrm{DMF}$; glassy carbon working electrode, glassy carbon rod counter electrode, $\mathrm{Ag} / \mathrm{AgCl}$ pseudoreference electrode; 100 $\mathrm{mV} / \mathrm{s}$ scan rate; referenced to internal ferrocene standard. Data previously reported. ${ }^{2}$

Table S19. Relative potential (E) and corrected (concentration and quasiharmonic) triple zeta free energies $([\mathrm{qG}])$ for the anionic carbonyl complexes $[\mathrm{Cr}(\mathrm{L})(\mathrm{CO})(\mathrm{DMF}) \mathrm{n}]^{-}(\mathrm{n}=$ $0,1)$, in $\mathrm{kcal} / \mathrm{mol}$. The entry in orange presented spin contamination.

\begin{tabular}{|c|c|c|c|}
\hline Species & $\mathbf{E}$ & $\mathbf{~} \mathbf{q G}]$ & $\left.<\mathbf{S}^{2}>\mathbf{( D Z} / \mathbf{T Z}\right)$ \\
\hline${ }_{0}^{2} \mathbf{C r}(\mathbf{C O})^{-1}+\mathrm{DMF}$ & 0.0 & 0.0 & $0.8061 / 0.8143$ \\
\hline${ }_{0}^{4} \mathbf{C r}(\mathbf{C O})^{-1}+\mathrm{DMF}$ & +5.2 & +3.8 & $3.7530 / 3.7530$ \\
\hline${ }_{0}^{6} \mathbf{C r}(\mathbf{C O})^{-1}+\mathrm{DMF}$ & +4.6 & +0.9 & $8.7502 / 8.7503$ \\
\hline${ }_{1}^{2} \mathbf{C r}(\mathbf{C O})^{-1}$ & -9.6 & +1.7 & $0.8764 / 0.8899$ \\
\hline${ }_{1}^{4} \mathbf{C r}(\mathbf{C O})^{-1}$ & -6.4 & +4.1 & $3.7547 / 3.7548$ \\
\hline${ }_{1}^{6} \mathbf{C r}(\mathbf{C O})^{-1}$ & -3.6 & +4.4 & $8.7503 / 8.7503$ \\
\hline
\end{tabular}

$$
P H N Z^{-}+{ }_{1}^{3} \operatorname{Cr}(\mathrm{CO}){ }^{0} \rightleftharpoons P H N Z+{ }_{0}^{2} \operatorname{Cr}(\mathrm{CO})^{-1}+D M F
$$

Eq S12: $\Delta[\mathrm{qG}]=13.6 \mathrm{kcal} / \mathrm{mol}$

$\mathrm{V}$ vs $\mathrm{PHNZ}^{-} / \mathrm{PHNZ}=-0.59 . \mathrm{V}$ vs Fc$+/ \mathrm{Fc}=-2.21$. Calibrated $\mathrm{V}$ vs $\mathrm{Fc}^{+} / \mathrm{Fc}=-2.09$. 


\section{Bicarbonate Formation}

Table S20. Relative potential (E) and corrected (concentration and quasiharmonic) triple zeta free energies $([\mathrm{qG}])$ for the transition states for $\mathrm{HCO}_{3}^{-}$formation from ${ }_{1}^{3} \mathrm{Cr}\left(\mathrm{CO}_{2} \mathbf{H}\right)^{-1}$ and $\mathrm{CO}_{2}$, in $\mathrm{kcal} / \mathrm{mol}$.

\begin{tabular}{|c|c|c|c|}
\hline Species & $\mathbf{E}$ & {$[q G]$} & $<\mathrm{S}^{2}>(\mathrm{DZ} / \mathrm{TZ})$ \\
\hline${ }_{0}^{3} \mathrm{TS6}^{-1}+\mathrm{DMF}$ & +19.5 & +19.4 & $2.0265 / 2.0278$ \\
\hline${ }_{0}^{3} \mathrm{TS}^{-1}+\mathrm{DMF}$ & +19.2 & +17.9 & $2.0150 / 2.0160$ \\
\hline${ }_{0}^{3} \mathrm{TS8}^{-1}+\mathrm{DMF}$ & +17.8 & +18.1 & $2.0094 / 2.0100$ \\
\hline${ }_{0}^{5} \mathrm{TS6}^{-1}+\mathrm{DMF}$ & +27.2 & +25.8 & $6.0004 / 6.0004$ \\
\hline${ }_{0}^{5} \mathrm{TS}^{-1}+\mathrm{DMF}$ & +28.0 & +25.0 & $6.0003 / 6.0003$ \\
\hline${ }_{0}^{5} \mathbf{T S 8}^{-1}+\mathrm{DMF}$ & +23.3 & +22.3 & 6.0004 / 6.0004 \\
\hline${ }_{1}^{3} \mathrm{TS6}^{-1}$ & +6.6 & +18.3 & $2.0318 / 2.0328$ \\
\hline${ }_{1}^{3} \mathrm{TS}^{-1}$ & +6.0 & +16.7 & $2.0207 / 2.0217$ \\
\hline${ }_{1}^{3} \mathrm{TS8}^{-1}$ & +3.5 & +16.0 & $2.0165 / 2.0175$ \\
\hline${ }_{1}^{5} \mathrm{TS6}^{-1}$ & +13.5 & +24.6 & $6.0003 / 6.0003$ \\
\hline${ }_{1}^{5} \mathrm{TS7}^{-1}$ & +13.6 & +23.9 & $6.0003 / 6.0003$ \\
\hline${ }_{1}^{5} \mathrm{TS8}^{-1}$ & +11.9 & +22.9 & $6.0003 / 6.0003$ \\
\hline
\end{tabular}



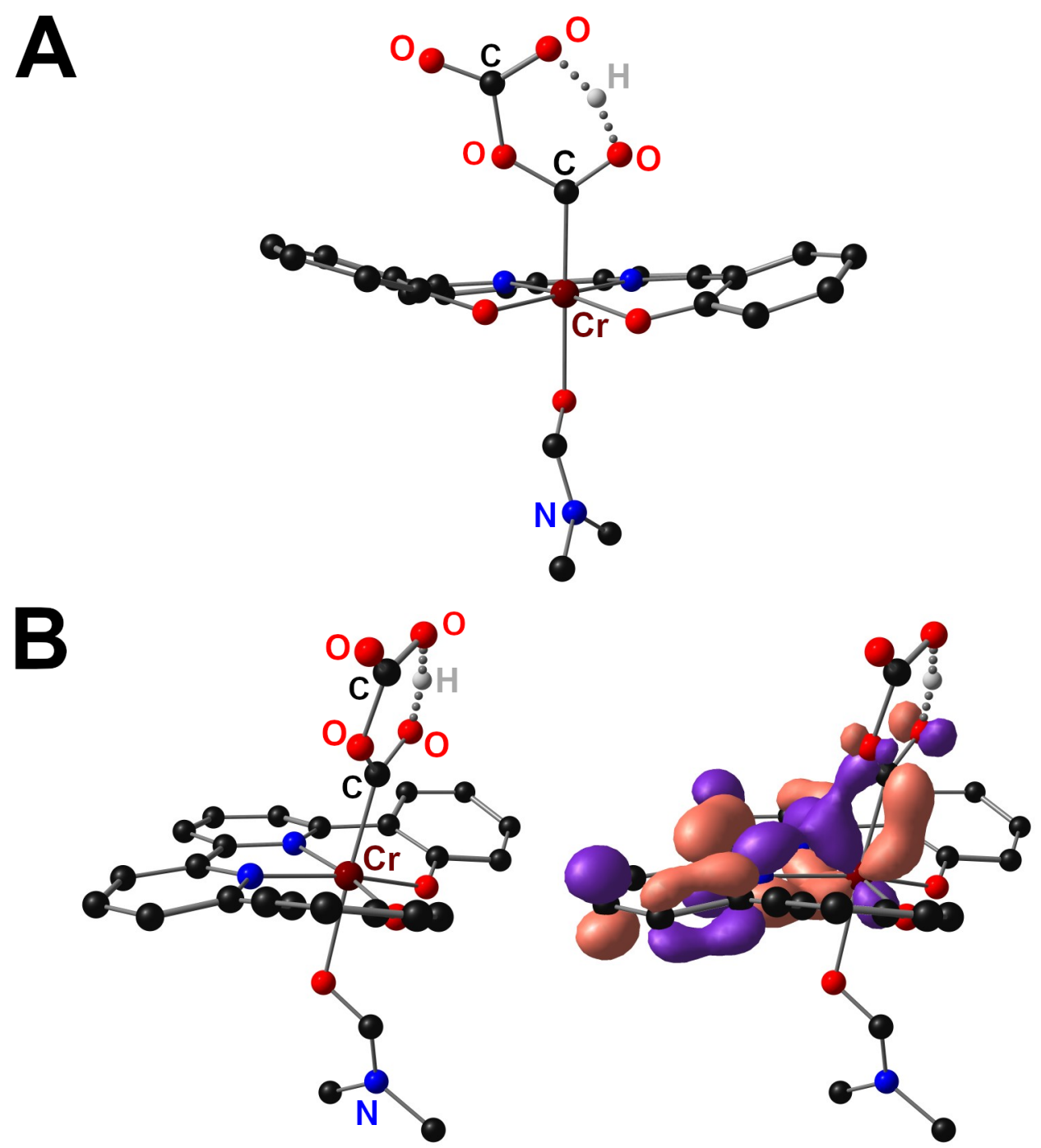

Figure S23. (A) Molecular geometry and (B) highest SOMO of ${ }_{1}^{3} \mathbf{T S 7}^{-1}$ for the conversion of ${ }_{1}^{3} \mathrm{Cr}\left(\mathrm{CO}_{2} \mathrm{HCO}_{2}\right)^{-1}$ into ${ }_{1}^{3} \mathrm{Cr}\left(\mathrm{CO}_{2} \mathrm{CO}_{2} \mathrm{H}\right)^{-1}$. For clarity, tBu groups and most $\mathrm{H}$ atoms have been omitted. 
A
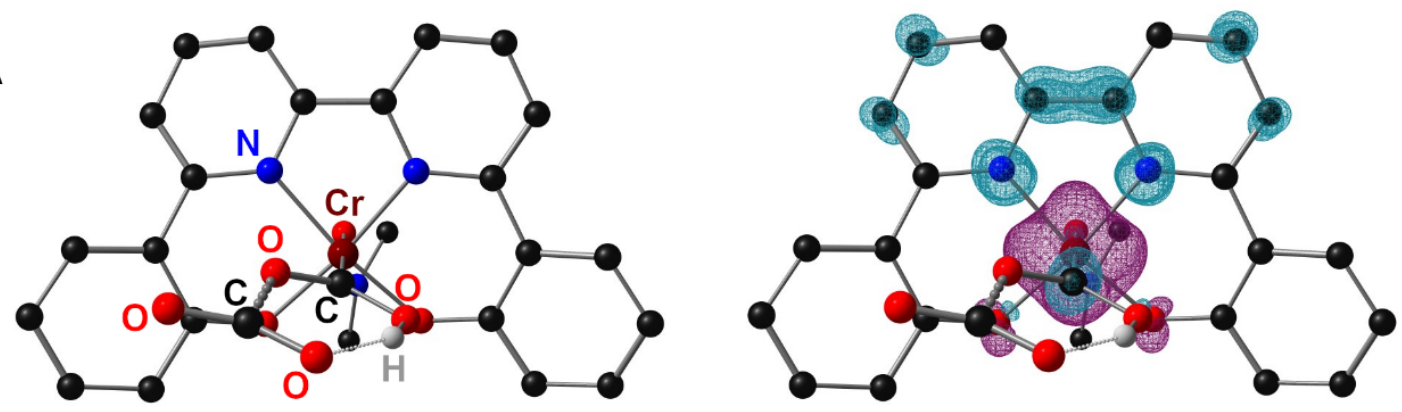

B
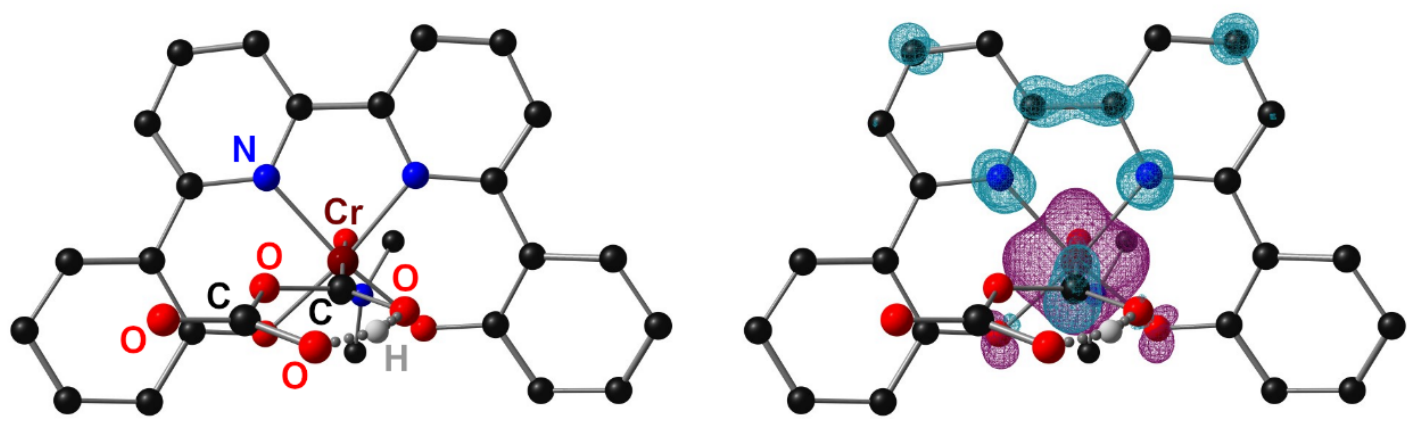

C
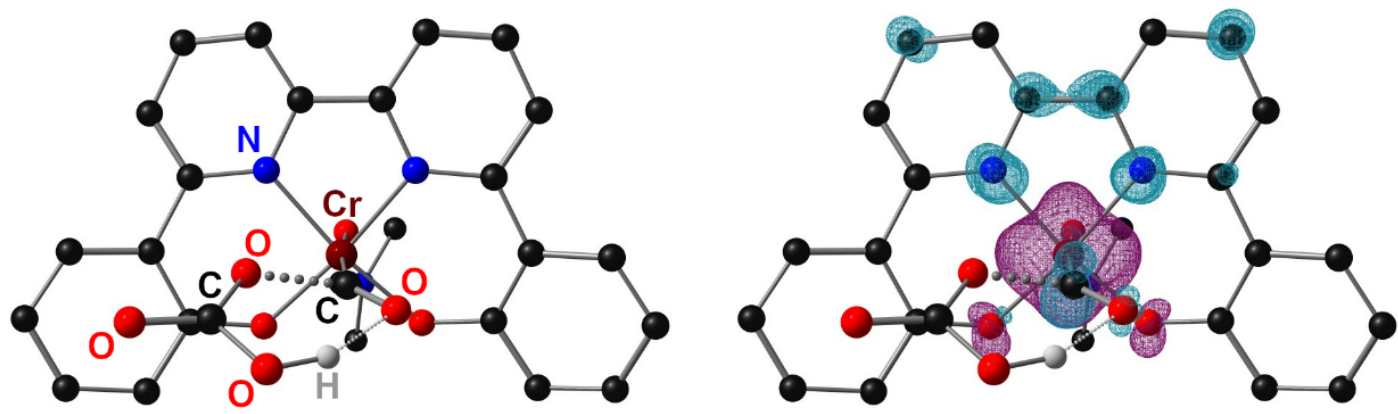

Figure S24. Spin density plots of (A) ${ }_{1}^{3}$ TS6 $^{-1}$,

(B) ${ }_{1}^{3} \mathrm{TS}^{-1}$ and (C) ${ }_{1}^{3} \mathrm{TS8}^{-1}$. For clarity, tBu groups and most $\mathrm{H}$ atoms have been omitted. 
A

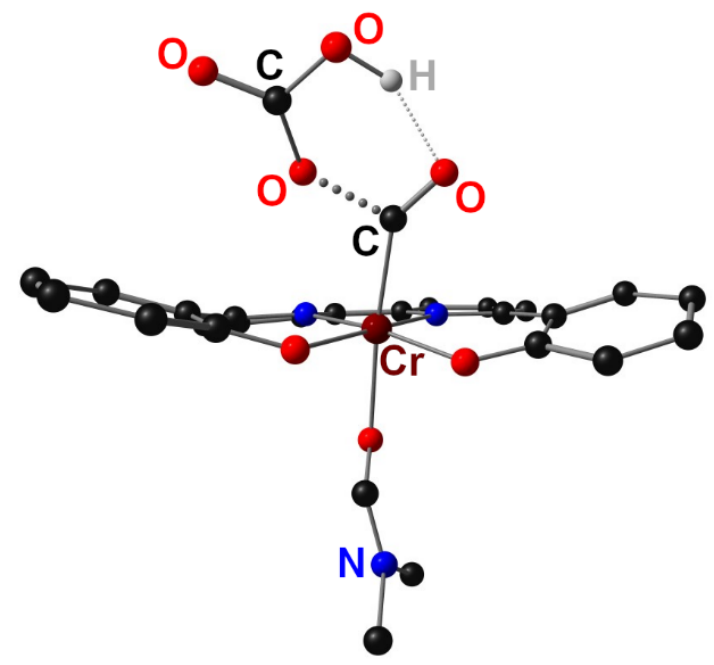

B
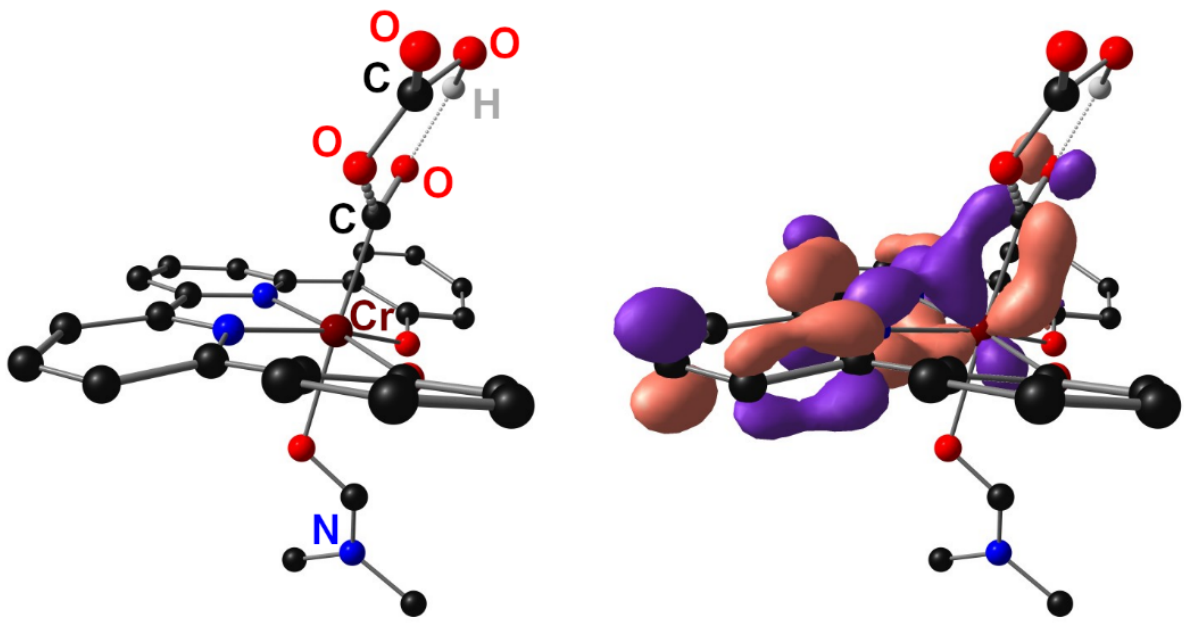

Figure S25. (A) Molecular geometry and (B) highest SOMO of ${ }_{1}^{3} \mathbf{T S 8}^{-1}$ for the conversion of ${ }_{1}^{3} \mathbf{C r}\left(\mathrm{CO}_{2} \mathrm{CO}_{2} \mathrm{H}\right)^{-1}$ into ${ }_{1}^{3} \mathrm{Cr}(\mathrm{CO}){ }^{0}$ and $\mathrm{HCO}_{3}{ }^{-}$. For clarity, tBu groups and most $\mathrm{H}$ atoms have been omitted.

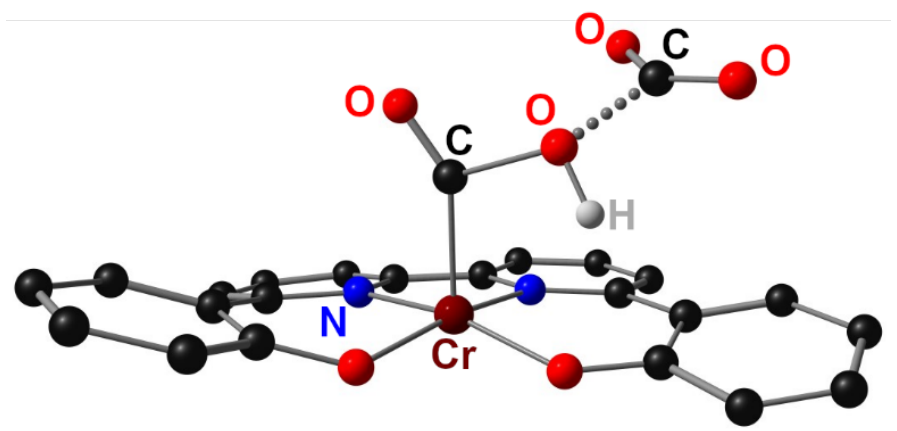

Figure S26. Molecular geometry of the transition state for the concerted pathway for bicarbonate formation in the triplet spin manifold $\left({ }_{0}^{3} \mathbf{T S 9}^{-1}\right)$. For clarity, tBu groups and most $\mathrm{H}$ atoms have been omitted. 


\section{Hydride Formation}

Table S21. Relative potential (E) and corrected (concentration and quasiharmonic) triple zeta free energies $([\mathrm{qG}])$ for the $\operatorname{Cr}(\mathrm{L})(\mathrm{H})(\mathrm{DMF})_{\mathrm{n}}(\mathrm{n}=0,1)$ species, in $\mathrm{kcal} / \mathrm{mol}$.

\begin{tabular}{|c|c|c|c|}
\hline Species & $\mathbf{E}$ & {$[\mathbf{q G}]$} & $<\mathbf{S}^{2}>(\mathbf{D Z} / \mathbf{T Z})$ \\
\hline${ }_{0}^{2} \mathbf{C r}(\mathbf{H}){ }^{0}+\mathrm{DMF}$ & +35.3 & +23.7 & $0.7836 / 0.7841$ \\
\hline${ }_{0}^{4} \mathbf{C r}(\mathbf{H})^{0}+\mathrm{DMF}$ & +14.6 & +2.6 & $3.7504 / 3.7504$ \\
\hline${ }_{1}^{4} \mathbf{C r}(\mathbf{H})$ & 0.0 & 0.0 & $3.7503 / 3.7503$ \\
\hline
\end{tabular}

$$
\begin{aligned}
& {[C r(L)]^{-}+P h O H+D M F \rightleftharpoons[C r(L)(H)(D M F)]+P h O^{-}} \\
& {[\mathrm{Cr}(L)]^{-}+2 \mathrm{PhOH}+\mathrm{DMF} \rightleftharpoons[\mathrm{Cr}(L)(H)(D M F)]+\left[(\mathrm{PhO})_{2} H\right]^{-}}
\end{aligned}
$$

Eq S14: $\Delta[\mathrm{qG}]=-7.2 \mathrm{kcal} / \mathrm{mol}$ 


\section{Ligand Protonation}

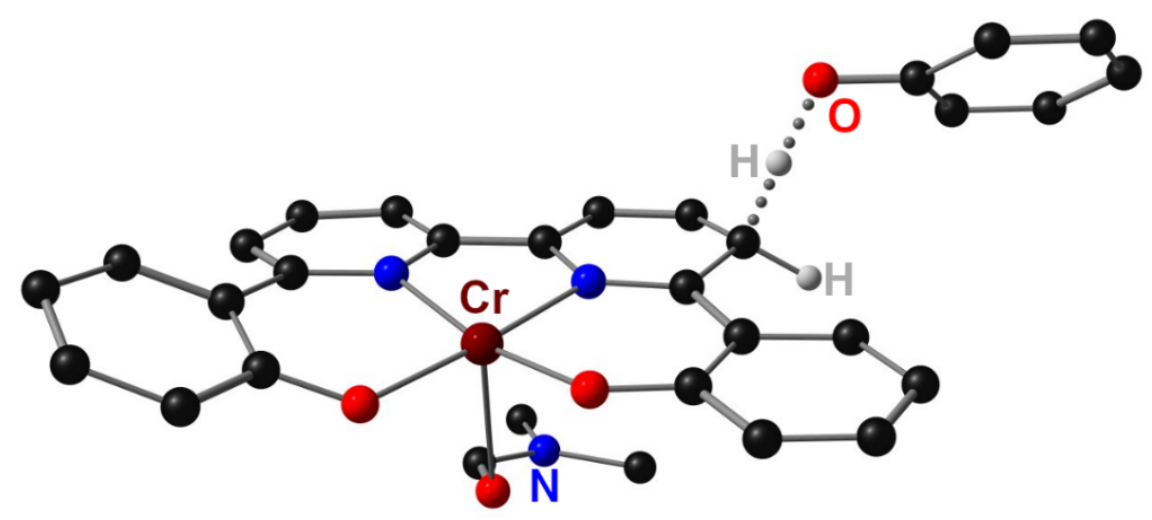

Figure S27. Molecular geometry of the transition state for the protonation of the 5position of the bpy backbone in the quartet spin state, ${ }_{1}^{4} \mathbf{T S 1 1}^{-1}$. For clarity, tBu groups and most $\mathrm{H}$ atoms have been omitted.

Table S22. Relative potential (E) and corrected (concentration and quasiharmonic) triple zeta free energies ([qG]) for the products resulting from protonation of the bpy backbone at the 5 position, in $\mathrm{kcal} / \mathrm{mol}$.

\begin{tabular}{|c|c|c|c|}
\hline Species & $\mathbf{E}$ & [qG] & $<\mathrm{S}^{2}>(\mathrm{DZ} / \mathrm{TZ})$ \\
\hline${ }_{0}^{4} \mathbf{b p y H}^{+}-5^{0}+\mathrm{DMF}$ & +14.2 & +1.0 & $3.8067 / 3.8065$ \\
\hline${ }_{0}^{6}$ bpyH $^{+}-5^{0}+$ DMF & +13.7 & +0.5 & $8.7505 / 8.7504$ \\
\hline${ }_{1}^{4} \mathrm{bpyH}^{+}-5^{0}$ & 0.0 & 0.0 & $3.7505 / 3.7504$ \\
\hline${ }_{1}^{6} \mathrm{bpyH}^{+}-5^{0}$ & 2.9 & +1.0 & $8.7504 / 8.7504$ \\
\hline
\end{tabular}

$$
\begin{gathered}
{[C r(L)]^{-}+P h O H+D M F} \\
\text { Eq S15: } \Delta[\mathrm{qG}]=+8.1 \mathrm{kcal} / \mathrm{mol} \\
{[\mathrm{Cr}(L)]^{-}+2 \mathrm{PhOH}+\mathrm{DMF} \rightleftharpoons[\operatorname{Cr}(\mathrm{LH})(\mathrm{DMF})]+\left[(\mathrm{PhO})_{2} \mathrm{H}\right]^{-}}
\end{gathered}
$$

Eq S16: $\Delta[\mathrm{qG}]=+2.9 \mathrm{kcal} / \mathrm{mol}$ 


\section{Three-electron reduced species}

Table S23. Relative potential (E) and corrected (concentration and quasiharmonic) triple zeta free energies $([\mathrm{qG}])$ for $[\mathrm{Cr}(\mathrm{L})]^{2-}$ species, in $\mathrm{kcal} / \mathrm{mol}$. Entries in red presented large spin contamination.

\begin{tabular}{|l|c|c|c|}
\hline Species & $\mathbf{E}$ & {$[\mathbf{q G}]$} & $\left.<\mathbf{S}^{2}>\mathbf{( D Z} / \mathbf{T Z}\right)$ \\
\hline${ }_{0}^{1} \mathbf{C r}^{-2}$ & +37.0 & +39.7 & $3.5799 / 3.7689$ \\
\hline${ }_{0}^{3} \mathbf{C r}^{-2}$ & +6.0 & +5.9 & $3.6219 / 3.6327$ \\
\hline${ }_{0}^{5} \mathbf{C r}^{-2}$ & 0.0 & 0.0 & $6.0014 / 6.0014$ \\
\hline
\end{tabular}

\section{Functional Testing}

Single point calculations with the PBE0-D3(BJ $)^{3}, \mathrm{~B}^{2} 7 \mathrm{D}^{4}$ and $\omega \mathrm{B} 97 \mathrm{XD}^{5}$ functionals were carried out on B3LYP-D3(BJ)-optimized geometries at the 6-311+G(2d,p) level of theory for representative steps of the cycle.

Table S24. Free energies of key steps in $\mathrm{kcal} / \mathrm{mol}$ and mean absolute difference (MAD) of the relative free energies versus B3LYP values.

\begin{tabular}{|c|c|c|c|c|}
\hline & $\begin{array}{c}\text { B3LYP- } \\
\text { D3(BJ) }\end{array}$ & PBE0-D3(BJ) & B97D & $\omega B 97 X D$ \\
\hline $\begin{array}{l}{ }_{0}^{4} \mathrm{Cr}^{-1}+\mathrm{PHNZ}- \\
{ }_{0}^{5} \mathrm{Cr}^{0}-\mathrm{PHNZ}^{-}\end{array}$ & +11.4 & +11.8 & +9.2 & +13.0 \\
\hline${ }_{0}^{4} \mathbf{C r}^{-1}+\mathrm{CO}_{2}$ & 0.0 & 0.0 & 0.0 & 0.0 \\
\hline${ }_{0}^{4} \mathbf{T S 3}^{-1}+\mathrm{DMF}$ & +10.0 & +12.3 & +4.8 & +15.9 \\
\hline${ }_{0}^{4} \mathrm{Cr}\left(\mathrm{CO}_{2}\right)^{-1}+\mathrm{DMF}$ & +4.7 & +10.4 & +1.5 & +8.8 \\
\hline $\begin{array}{c}{ }_{1}^{3} \mathrm{Cr}\left(\mathrm{CO}_{2} \mathbf{H}\right)^{-1}+ \\
\mathrm{PhOH}\end{array}$ & 0.0 & 0.0 & 0.0 & 0.0 \\
\hline${ }_{1}^{3} \mathrm{TS5}^{-1}$ & +10.5 & +15.4 & +7.2 & +16.1 \\
\hline${ }_{0}^{5} \mathrm{TS5}^{-1}+\mathrm{DMF}$ & +11.1 & +15.3 & +8.5 & +11.7 \\
\hline MAD & & 3.5 & 3.3 & 3.6 \\
\hline
\end{tabular}




\section{Determination of TOF from Preparative Electrolysis}

The integrated expression of current for a homogeneous electrocatalytic response (considering an application of steady-state conditions to the substrate) has been solved previously ${ }^{6}$ :

$$
\frac{i}{F A}=\frac{n_{c a t}^{\sigma}[c a t] \sqrt{\left(k_{o b s} C_{A}^{0} D_{c a t}\right)}}{1+\exp \left[\frac{F}{R T}\left(E_{a p p}-E_{1 / 2}\right)\right]}
$$

where $i$ is the average current (Amps) specific to the reaction product of interest, $F$ is Faraday's constant $\left(96485 \mathrm{C} \mathrm{mol}^{-1}\right), A$ is the area of the electrode $\left(\mathrm{cm}^{2}\right), n_{c a t}^{\sigma}$ is the number of electrons in the catalytic process (2) with $\sigma=1$ under the assumption that all electrons are delivered to the catalyst by the electrode ( $\sigma=0.5$ corresponds to the case where homogeneous electron transfer occurs between catalyst molecules in solution), [cat] is the concentration of the catalyst $\left.(\mathrm{mol} \mathrm{cm})^{-3}\right), k_{o b s}$ is the apparent turnover frequency $\left(\mathrm{s}^{-1}\right), C_{A}^{0}$ is the concentration of $\mathrm{CO}_{2}$ saturated in DMF $\left(\mathrm{mol} \mathrm{cm}{ }^{-3}\right), D_{\text {cat }}$ is the diffusion coefficient of the catalyst $\left(\mathrm{cm}^{2} \mathrm{~s}^{-1}\right), R$ is the ideal gas constant (Joule $\mathrm{mol}^{-1} \mathrm{~K}^{-}$ $\left.{ }^{1}\right), T$ is the temperature $(\mathrm{K}), E_{a p p}$ is the applied potential during preparative electrolysis $(\mathrm{V})$, and $E_{1 / 2}$ is the standard potential of the catalyst (V).

and

$$
\frac{i}{A}=J=C O \text { specific current density }
$$

Substituting and rearranging the first expression to solve for $k_{\mathrm{obs}}$

$$
k_{o b s}=\frac{J^{2}\left(1+\exp \left[\frac{F}{R T}\left(E_{a p p}-E_{1 / 2}\right)\right]\right)^{2}}{F^{2}\left(n_{c a t}^{\sigma}[c a t]\right)^{2} C_{A}^{0} D_{c a t}}
$$

with $k_{o b s}$ in hand, the TOF can be expressed for a given potential according to the following relationship

$$
T O F=\frac{k_{o b s}}{1+\exp \left[\frac{F}{R T}\left(E_{a p p}-E_{1 / 2}\right)\right]}
$$

Previously reported (data from $\mathrm{Ref}^{2}$ ) parameters for electrocatalysis mediated by

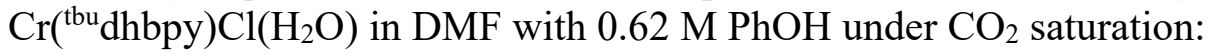

- Average recorded CO specific current density: $-0.00032963 \mathrm{~A} \mathrm{~cm}^{-2}$

- Applied potential $\left(E_{a p p}\right):-2.1 \mathrm{~V}$ vs $\mathrm{Fc}^{+} / \mathrm{Fc}$

- $\mathrm{E}_{1 / 2}$ catalyst: $-1.95 \mathrm{~V}$ vs $\mathrm{Fc}^{+} / \mathrm{Fc}$

- Temperature: $298.15 \mathrm{~K}$

- $\quad\left[\mathrm{CO}_{2}\right]: 2.3 \times 10^{-4} \mathrm{~mol} \mathrm{~cm}^{-3}$

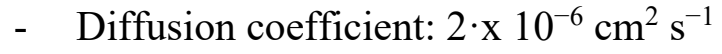

- Catalyst concentration: $5.8 \times 10^{-7} \mathrm{~mol} \mathrm{~cm}^{-3}$ 


\section{Results from applying the energetic span model:}

For consistency with the main text, absolute energy values relative to the energy of the pre-catalyst species ${ }_{1}^{4} \mathbf{C r}(\mathbf{C l}){ }^{0}$ were used in the input as shown here. Adding 13.7 $\mathrm{kcal} / \mathrm{mol}$ to all values in the input file produces a catalytic cycle beginning at 0.0 , but has no impact on the predicted TOF or TOF-determining transition state described in the main text in a comparable simulation. It is worth emphasizing that the electrocatalytic cycle begins and ends at the four-coordinate neutral species ${ }_{0}^{5} \mathrm{Cr}^{0}$ : all preceding species computed and described in the main text are pre-catalytic and will not contribute.

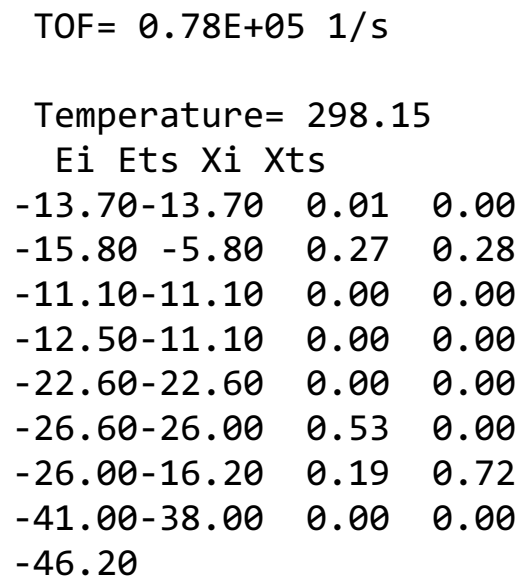

${ }^{1}$ Roy, L. E.; Jakubikova, E.; Guthrie, M. G.; Batista, E. R. Calculation of One-Electron Redox Potentials Revisited. Is It Possible to Calculate Accurate Potentials with Density Functional Methods? J. Phys. Chem. A 2009, 113, 6745.

2 Hooe, S. L.; Dressel, J. M.; Dickie, D. A.; Machan, C. W. Highly Efficient Electrocatalytic Reduction of $\mathrm{CO}_{2}$ to $\mathrm{CO}$ by a Molecular Chromium Complex. ACS Catal. 2020, 10, 1146.

${ }^{3}$ Perdew, J. P.; Burke, K.; Ernzerhof, M. Generalized Gradient Approximation Made Simple. Phys. Rev. Lett. 1996, 77, 3865.

${ }^{4}$ Grimme, S. Semiempirical GGA-type density functional constructed with a long-range dispersion correction. J. Comput. Chem. 2006, 27, 1787.

${ }^{5}$ Chai, J.-D.; Head-Gordon, M. Long-range corrected hybrid density functionals with damped atom-atom dispersion corrections. Phys. Chem. Chem. Phys. 2008, 10, 6615.

${ }^{6}$ a) Costentin, C.; Drouet, S.; Robert, M.; Savéant, J.-M. Turnover Numbers, Turnover Frequencies, and Overpotential in Molecular Catalysis of Electrochemical Reactions. Cyclic Voltammetry and Preparative-Scale Electrolysis. J. Am. Chem. Soc. 2012, 134, 11235; b) Costentin, C.; Drouet, S.; Robert, M.; Savéant, J.-M. Correction to Turnover Numbers, Turnover Frequencies, and Overpotential in Molecular Catalysis of Electrochemical Reactions. Cyclic Voltammetry and Preparative-Scale Electrolysis. $J$. Am. Chem. Soc. 2012, 134, 19949. 